\title{
An Approach for the Impact Dynamic Modeling and Simulation of Planar Constrained Metamorphic Mechanism
}

\author{
Yanyan Song, ${ }^{1,2}$ Boyan Chang, ${ }^{1,2}$ Guoguang Jin (iD, ${ }^{1,2}$ Zhan Wei, ${ }^{1,2}$ and Bo Li ${ }^{1}$ \\ ${ }^{1}$ Shool of Mechanical Engineering, Tiangong University, Tianjin 300387, China \\ ${ }^{2}$ Tianjin Key Laboratory of Advanced Mechatronics Equipment Technology, Tianjin 300387, China
}

Correspondence should be addressed to Guoguang Jin; jinguoguang@tiangong.edu.cn

Received 31 March 2020; Revised 3 June 2020; Accepted 6 June 2020; Published 5 August 2020

Academic Editor: Dr Mahdi Mohammadpour

Copyright (c) 2020 Yanyan Song et al. This is an open access article distributed under the Creative Commons Attribution License, which permits unrestricted use, distribution, and reproduction in any medium, provided the original work is properly cited.

This paper studied the impact dynamic modeling of the planar constrained metamorphic mechanism (PCMM) during configuration transformation. Based on the dynamic theory of the multi-rigid-body system and the coefficient of restitution equation, a new method for dynamic modeling of PCMM considering impact motions generated by configuration transformation is presented, which can be treated as a theoretical foundation for performance design and dynamic control. Firstly, the topology theory based on the impact motion can be classified as the stable impact motion and the mobile impact motion, which is the prerequisite for dynamic modeling and simulation. Secondly, the stable and mobile impact dynamic models for PCMM are established according to the dynamic theory of the multi-rigid-body system. Then, using these models, the corresponding impulse solving models are deduced combining with the coefficient of restitution equation. Finally, the examples of the stable impact motion and the mobile impact motion are respectively given, and the configuration-complete dynamic simulations are carried out. By comparing with the dynamic models without considering the impact motion, the dynamic characteristics of PCMM are analyzed. The theory and method proposed in this paper can be also applied in general planar robotic systems to deal with the problem of internal collision dynamics.

\section{Introduction}

Metamorphic mechanisms are members of the class of mechanisms that are able to change their configurations sequentially to meet different requirements [1]. Since the proposal of metamorphic mechanism in 1998 [2], it has been widely applied to various fields of engineering, such as bionic joint mechanism [3, 4], extendable/foldable flexible spacecraft mechanism [5], automated laying mechanism [6, 7], robot mechanism [8], and space metamorphic parallel mechanism [9]. The applications of the current metamorphic mechanisms are mainly based on task-orientated constrained metamorphic mechanisms [10], which have become the focus of analysis and research in the field of mechanism.

Based on the division method of the metamorphic mode, the metamorphic mechanisms have many ways to achieve configuration transformation [11]. Constrained metamorphic mechanism is a kind of mechanism, which realizes configuration transformation by using geometric constraints and/or force constraints to reduce the number of degrees of freedom (DOFs) of a multi-DOF metamorphic mechanism to the number of driving links [12]. In the course of movement and work, the constraint types and the characteristics of metamorphic joints will change along with the continuous transformation of configurations. The internal impact is generated by configuration transformation, whose characteristics are very brief duration, high force levels reached, rapid dissipation of energy, and large accelerations and decelerations present [13]. In severe cases, it will directly affect stability, reliability, and safety of the system. It can be said that the characteristics of impact motion during configuration transformation directly determine whether the system can work safely and steadily or not in the constrained metamorphic mechanisms. Therefore, it is necessary to study the impact characteristics of the constrained metamorphic mechanism during configuration transformation. 
Constrained metamorphic mechanism belongs to the multibody system; furthermore, the impact motion of the constrained metamorphic mechanism can be regarded as a typical collision dynamic problem of the multibody system with variable topological structures. The research on collision dynamic modeling methods of the multibody system can be divided into the following categories: impulse-momentum method, continuous model, and Lagrange multiplier method. In the impulse-momentum method, the colliding bodies are assumed to be rigid and no deformation occurs at the impact location. Duan and Zhang [14] introduced the concept of impact potential energy and investigated the rigid-flexible coupling dynamics of a radially rotating flexible beam with impact. Jin et al. [15] proposed a method based on the impulse principle and the Gauss minimum constraint method to determine the system state variables after contact collision. Dong and Chen [16] analyzed the impact dynamics and the effect between the space manipulator end-effector and the satellite of the capture process with the momentum impulse method combining the momentum conservation principle. Deng et al. [17] used the impulse-momentum equation involving the restitution coefficient of the system to establish the impact dynamic model which can extrapolate the impact momentum on the drive component. Choi et al. [18] derived an analytical model of the restitution coefficient for the 3 DOF finger model colliding with a wall and showed that COR varied according to the colliding velocity and its contact area through the experiment. Although the impulse-momentum method has the advantages of high calculation efficiency and intuitionistic, it cannot calculate the collision process. In the continuous model, the impact-induced force is a function of the relative penetration of two bodies into each other. Machado et al. [19] presented a general and comprehensive study of some of the most relevant compliant contact force models for multibody system dynamics. Also, two simple planar multibody systems that included contact-impact scenarios were considered as examples of application to demonstrate the similarities of and differences between the contact force models used throughout this work. Bhalerao and Anderson [20] presented a complementarity-based recursive scheme to model intermittent contact for flexible multibody systems. Ahmadizadeh et al. [21] dynamically modeled the phenomenon of multiple impact-contacts based on the continuous contact force model for an open kinematic chain with rigid links and revolute joints. Korayem et al. [22, 23] studied the modeling of various contact theories for application in the manipulation of different biological micro/nanoparticles. Flores and Ambrósio [24] used a contact force model in contact-impact analysis in multibody dynamics and explicitly accounted for the deformation of the bodies during the impact process. The continuous model can objectively reflect the collision process, but the calculation form of collision force and the selection of relevant parameters are relatively difficult. Compared with the continuous model, the Lagrange multiplier method avoids the uncertainty caused by parameter selection and improves the reliability of the model. But, it is not easy to obtain a more general method to solve the velocity increments. To bridge the gap between accuracy and efficiency in the dynamic simulation of a flexible multibody system with contacts/impacts, Wang et al. [25] modeled the impact region using the finite element method. In addition, Hurmuzlu and Marghitu [26] studied the rigid body collision of planar, kinematic chains with an external surface while in contact with other surfaces. A. M. Shafei and H. R. Shafei [27] presented a systematic procedure for the dynamic modeling of a closed-chain robotic system in both the flight and impact phases. Gattringer et al. [28] established an efficient dynamic model for rigid multibody systems with contact and impact by a recursive procedure. The above research studies are mainly aimed at the dynamic response of the multibody system colliding with its working environments and other multibody systems.

In this work, the impact dynamics of the constrained metamorphic mechanism during configuration transformation is mainly studied along with the impact dynamics between two internal components of the multibody system. Wang et al. [29] considered the effects of material parameters, control parameters, and internal impact excitation on dynamic performance of the mechanism system. Bruzzone and Bozzini [30] designed a novel microassembly system and tested the grasping stability of the metamorphic fingertips. Song et al. [31] studied the change of the $6 \mathrm{R}$ metamorphic mechanism from an unconstrained condition to a geometrically constrained condition to accomplish the transformation between different screw systems. Ye et al. [32] analyzed the configuration changes of the reconfigurable limb associated with the three distinct phases of the PMM (planar metamorphic mechanism) and identified the constraints exerted by the reconfigurable limb in various configurations. Until now, the configuration-complete dynamic model of the metamorphic mechanism has been mainly established by incorporating both the topological structure change and the subconfiguration dynamic model [33], ignoring the impact motion generated by configuration transformation. It should be noticed that in order to keep favorable dynamic performance, the impact motion generated by configuration transformation should be taken into consideration in the dynamic modeling.

Therefore, to describe the influence of impact motion on the system's stability when the configuration changes, the impact dynamics during configuration transformation need to be studied. In this paper, taking PCMM as an example, the dynamic models of its stable and mobile impact motions are established based on the topology theory and multibody system dynamics, and then the corresponding impulse solving models are deduced by combining the coefficient of restitution equation. Further, two examples of PCMM considering the impact motions generated by configuration transformation are given to illustrate the effect of impact motions. Meanwhile, the validity of the proposed method is verified.

\section{Dynamic Analysis}

The working process of PCMM can be roughly divided into the subconfiguration movement stage and the instantaneous configuration transformation stage. Therefore, the configuration- 
complete dynamic equation of PCMM considering the impact motion during configuration transformation will be set up.

\subsection{Dynamic Analysis in Subconfiguration Movement Stage.} Under any working stage or working condition $p$, the corresponding topological structure of PCMM is called the $p$-th configuration or configuration $p$. PCMM in any configuration $p$ can be regarded as a conventional mechanism. Suppose the DOF of PCMM in configuration $p$ is $n$ and the independent generalized coordinate of PCMM is ${ }^{p} \mathbf{q}=\left[{ }^{p} q_{1},{ }^{p} q_{2}, \ldots,{ }^{p} q_{n}\right]^{\mathrm{T}} \in \mathbf{R}^{n \times 1}$. Ignoring the influence of external disturbance such as friction, the dynamic equation of PCMM with any configuration $p$ can be written by the Newton-Euler equation as a vector form:

$$
{ }^{p} \mathbf{D}^{p} \ddot{\mathbf{q}}+{ }^{p} \mathbf{H}={ }^{p} \boldsymbol{\tau}_{a}+{ }^{p} \boldsymbol{\tau}_{c},
$$

where $^{p} \ddot{\mathbf{q}} \in \mathbf{R}^{n \times 1}$ is the generalized acceleration vector, ${ }^{p} \mathbf{D} \in \mathbf{R}^{n \times 1}$ is the inertia matrix which is symmetric positive definite, ${ }^{p} \mathbf{H} \in \mathbf{R}^{n \times 1}$ is the gravity, the Coriolis, and the centripetal force vector, ${ }^{p} \boldsymbol{\tau}_{a} \in \mathbf{R}^{n \times 1}$ is the generalized force vector, and ${ }^{p} \boldsymbol{\tau}_{c} \in \mathbf{R}^{n \times 1}$ is the generalized elastic and other generalized force vector.

\subsection{Dynamic Analysis in Instantaneous Configuration} Transformation Stage. For PCMM with $c$ configurations, it is assumed that configuration $p(1 \leq p \leq c-1)$ is any configuration of PCMM. When PCMM is changed from configuration $p$ to configuration $p+1$, the impact motion occurs inside the system. There are mainly two situations as follows:

(1) The moving component is merged with the frame

(2) The moving component is merged with another moving component

To establish the instantaneous impact dynamic equation for configuration transformation of PCMM, the definitions are as follows.

When PCMM is changed from one configuration to the next,

(1) According to the way of configuration transformation, if the moving component is merged with the frame, the impact motion generated by configuration transformation is named the stable impact motion and the generated internal impulse is named the stable impulse in this paper. Meanwhile, the dynamic equation of the system is named the stable impact dynamic equation.

(2) Similarly, if the moving component is merged with another moving component, the impact motion generated by configuration transformation is named the mobile impact motion and the generated internal impulse is named the mobile impulse. At this time, the dynamic equation of the system is named the mobile impact dynamic equation.

It should be further pointed out that since the impact motion is generated instantaneously during the configuration transformation of PCMM, the impact dynamics is only studying the system state at a certain moment and not the system state throughout the time history.

2.2.1. Dynamic Analysis of the Stable Impact Motion. In this section, a dynamic model is established, which describes the dynamics of PCMM with a stable impact motion. In order to establish a complete theoretical system of impact dynamics of PCMM, it is very important to choose a reasonable topology structure. In this paper, it is assumed that the two adjacent components of PCMM are connected by rotating hinges, and the topology structure of PCMM can be considered as a tree system, as shown in Figure 1.

Assuming that the impact motion occurs instantaneously, the impact points are defined as ${ }^{p} X_{0}$ and ${ }^{p} X_{1}$ on the frame $B_{0}$ and the moving component $B_{1}$, respectively. When the moving component $B_{1}$ and the frame $B_{0}$ merge, the stable impact motion occurs, and there are equal and opposite action and reaction forces at the impact points ${ }^{p} X_{0}$ and ${ }^{p} X_{1}$. It is sometimes necessary to get more acquainted with the impact force of metamorphic components and the ideal constraint force of metamorphic joints for stability analysis of configuration transformation.

When the configuration of PCMM changes, the impulse generated by configuration transformation of the mechanism is zero [34]. However, when the moving component merges with the frame or the fixed base, the impulse acting on the moving component is not zero. To solve the impulse acting on the moving component $B_{1}$, the rotating joint $O_{1}$ connecting the moving component $B_{1}$ and the frame $B_{0}$ can be released based on multibody systems dynamics $[35,36]$, and the stable impact motion system can be transformed into the multibody system $f$ which is only subject to ideal constraints.

For the cutting of joints in the PCMM, that is to remove partial constraints in the system. The redundant joints removed are also called the cutoff joints. If the multibody system $f$ is equivalent to the original stable impact motion system in dynamics, the constraint force acting on the adjacent components by the cutoff joint must be considered as an additional external force applied to the multibody system $f$. Therefore, based on the Newton-Euler equation, a dynamic equation of the multibody system $f$ is established, and the stable impact dynamic equation is established combining the constraint equation of the cutoff joint $O_{1}$. And, the stable impact force of the metamorphic component $B_{1}$ and the ideal constraint force of metamorphic joint $O_{1}$ can be calculated by the stable impact dynamic equation. The detailed derivation process is as follows.

Assume that the generalized position vector of arbitrary configuration $p$ obtained by the cutting off joint $O_{1}$ is ${ }^{p} q_{f} \in \mathbf{R}^{n_{f} \times 1}$ and $n_{f}$ is the number of DOFs in the multibody system $f$. Then, position of the impact point ${ }^{p} X_{1}$ can be written separately as expressed as a function with a generalized coordinate ${ }^{p} q_{f}$ as

$$
{ }^{p} X_{1}={ }^{p} \boldsymbol{\varphi}_{f}\left({ }^{p} q_{f}\right) .
$$

According to the kinematic relationship of the multibody system $f$, it is easy to obtain the following kinematic relationship between the velocity of the impact point ${ }^{p} X_{1}$ on 


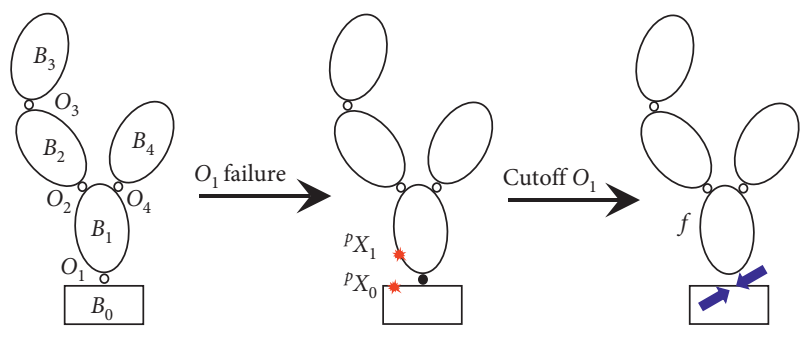

Figure 1: The stable impact motion system.

the moving component $B_{1}$ and the generalized velocity vector ${ }^{p} \dot{\mathbf{q}}_{f}$ :

$$
{ }^{p} \dot{X}_{1}={ }^{p}\left[\mathbf{J}_{q_{f}}^{v}\right]{ }^{p} \dot{\mathbf{q}}_{f},
$$

where

$$
p\left[\mathbf{J}_{q_{f}}^{v}\right]=\frac{\partial\left({ }^{p} \boldsymbol{\varphi}_{f}\right)}{\partial\left({ }^{p} \mathbf{q}_{f}\right)^{\mathrm{T}}} .
$$

When the system satisfies the conditions of configuration transformation ${ }^{p} X_{1}={ }^{p} X_{0}$, the stable impact force ${ }^{p} F_{s}$ acting on the impact points ${ }^{p} X_{1}$ and ${ }^{p} X_{0}$ is immediately generated. According to the Newton-Euler equation, the multibody system $f$ with the stable impact motion is subject to constraint forces at the cutoff joint and stable impact forces in addition to driven and elastic forces. Therefore, the dynamic equation of the multibody system $f$ with the stable impact motion can be established as

$$
{ }^{p} \mathbf{D}_{f}{ }^{p} \ddot{\mathbf{q}}_{f}+{ }^{p} \mathbf{H}_{f}={ }^{p} \boldsymbol{\tau}_{a f}+{ }^{p} \boldsymbol{\tau}_{c f}+{ }^{p} \boldsymbol{\tau}_{\text {cut } f}+{ }^{p} \boldsymbol{\tau}_{s f},
$$

where ${ }^{p} \ddot{\mathbf{q}}_{f}$ is the generalized acceleration vector of the multibody system $f,{ }^{p} \mathbf{D}_{f}$ is the inertia matrix of the multibody system $f,{ }^{p} \mathbf{H}_{f}$ is the gravity, the Coriolis, and centripetal force vector of the multibody system $f,{ }^{p} \boldsymbol{\tau}_{a f}$ is the generalized force vector of the multibody system $f,{ }^{P} \boldsymbol{\tau}_{c f}$ is the generalized elastic and other generalized force vector of the multibody system $f,{ }^{p} \boldsymbol{\tau}_{\text {cut } f}$ is the generalized constraint force vector of the cutoff joint $O_{1}$, and ${ }^{p} \boldsymbol{\tau}_{s f}$ is the generalized stable impact force vector.

According to the principle of virtual work, the generalized constraint force vector and the generalized stable impact force vector can be respectively expressed as

$$
\begin{gathered}
{ }^{p} \boldsymbol{\tau}_{\text {cut } f}=p\left[\mathbf{J}_{q_{f}}^{\text {cut }}\right]^{\mathrm{T}}{ }^{p} F_{\text {cut } f}, \\
{ }^{p} \boldsymbol{\tau}_{s f}=p\left[\mathbf{J}_{q_{f}^{v}}^{v}\right]^{\mathrm{T}}{ }^{p} F_{s},
\end{gathered}
$$

where ${ }^{P}\left[\mathbf{J}_{q_{f}}^{\text {cut }}\right]$ is the first-order kinematic influence coefficient of the velocity vector of the cutoff joint $O_{1}$ in the inertial coordinate system with respect to the generalized velocity in the multibody system $f$ and ${ }^{p} F_{\text {cut } f}$ is the constraint force at the cutoff joint $O_{1}$.

According to the Newton-Euler equation, the constraint force at the cutoff joint $O_{1}$ can be expressed as the form of generalized acceleration vector ${ }^{p} \ddot{\mathbf{q}}$ in the stable impact motion system:

$$
{ }^{p} F_{\text {cut } f}={ }^{p} \mathbf{D}_{\text {cut }}{ }^{p} \ddot{\mathbf{q}}+{ }^{p} \mathbf{H}_{\text {cut } f},
$$

where ${ }^{p} \mathbf{D}_{\text {cut }}$ is the coefficient matrix of ${ }^{p} F_{\text {cut } f}$ with respect to ${ }^{p} \ddot{\mathbf{q}}$ and ${ }^{p} \mathbf{H}_{\text {cut } f}$ is a column matrix.

According to the kinematic constraint equation at the cutoff joint $O_{1}$, that is, ${ }^{p} \ddot{\mathbf{q}} \subset{ }^{p} \ddot{\mathbf{q}}_{f}$. The constraint force at the cutoff joint $O_{1}$ can be expressed as

$$
{ }^{p} F_{\text {cut } f}={ }^{p} \mathbf{D}_{\text {cut } f}{ }^{p} \ddot{\mathbf{q}}_{f}+{ }^{p} \mathbf{H}_{\text {cut } f},
$$

where ${ }^{p} \mathbf{D}_{\text {cut } f}$ is the coefficient matrix of ${ }^{p} F_{\text {cut } f}$ with respect to ${ }^{p} \ddot{\mathbf{q}}_{f}$.

Combining equations (5)-(7) and (9), the stable impact dynamic equation of PCMM can be derived as

$$
\begin{gathered}
{ }^{p} \mathbf{D}_{f}{ }^{p} \ddot{\mathbf{q}}_{f}+{ }^{p} \mathbf{H}_{f}={ }^{p} \boldsymbol{\tau}_{c f}+{ }^{p} \boldsymbol{\tau}_{c f}+{ }^{p}\left[\mathbf{J}_{q_{f}}^{\mathrm{cut}}\right]^{\mathrm{T}}{ }^{p} \mathbf{D}_{\mathrm{cut} f}{ }^{p} \ddot{\mathbf{q}}_{f} \\
{ }^{p}\left[\mathbf{J}_{q_{f}}^{\mathrm{cut}}\right]^{T}{ }^{p} \mathbf{H}_{\mathrm{cut} f}+{ }^{p}\left[\mathbf{J}_{q_{f}}^{v}\right]^{T}{ }^{p} F_{s} .
\end{gathered}
$$

\subsubsection{Dynamic Analysis of the Mobile Impact Motion. As} shown in Figure 2, when the mobile impact motion occurs, the moving component $B_{2}$ merges with another moving component $B_{3}$, and there are equal and opposite action and reaction forces at the impact points ${ }^{p} X_{2}$ and ${ }^{p} X_{3}$. In order to obtain the mobile impact force of metamorphic components and the ideal constraint force of metamorphic joints, the rotating joint $\mathrm{O}_{3}$ between the moving components $B_{2}$ and $B_{3}$ is cut off based on multibody system dynamics $[35,36]$. Then, the mobile impact motion system is divided into two multibody systems with external impacts, which are respectively labeled as $f_{1}$ and $f_{2}$, and the dynamic equations of the multibody systems $f_{1}$ and $f_{2}$ can be established.

In order to make the divided multibody systems $f_{1}$ and $f_{2}$ dynamically equivalent to the mobile impact motion system, the constraint force acting on the adjacent components by the cutting off joint $\mathrm{O}_{3}$ must be regarded as an additional external force applied to the two multibody systems $f_{1}$ and $f_{2}$.

It is assumed that the generalized position vectors of the multibody systems $f_{1}$ and $f_{2}$ are ${ }^{p} \mathbf{q}_{f_{1}} \in \mathbf{R}^{n_{f_{1}} \times 1}$ and ${ }^{p} \mathbf{q}_{f_{2}} \in \mathbf{R}^{n_{f_{2}} \times 1}$, respectively, and $n_{1}$ and $n_{2}$ are the number of DOFs of the multibody systems $f_{1}$ and $f_{2}$, respectively. Then, positions of the impact points ${ }^{p} X_{2}$ and ${ }^{p} X_{3}$ can be written separately as expressed as functions with generalized coordinates ${ }^{p} \mathbf{q}_{f_{1}}$ and ${ }^{P} \mathbf{q}_{f_{2}}$ as

$$
\begin{aligned}
& { }^{p} X_{2}={ }^{p} \boldsymbol{\varphi}_{f_{1}}\left({ }^{p} \mathbf{q}_{f_{1}}\right), \\
& { }^{p} X_{3}={ }^{p} \boldsymbol{\varphi}_{f_{2}}\left({ }^{p} \mathbf{q}_{f_{2}}\right) .
\end{aligned}
$$

According to the kinematic relationship of the multibody systems $f_{1}$ and $f_{2}$, it is easy to obtain the following kinematic relationship between the velocity of the impact point ${ }^{p} X_{2}$ on the moving component $B_{2}$ in the inertial coordinate system and the generalized velocity vector ${ }^{p} \mathbf{q}_{f_{1}}$ and the velocity of the impact point ${ }^{p} X_{3}$ on the moving component $B_{3}$ in the inertial coordinate system and the generalized velocity vector ${ }^{P} \mathbf{q}_{f_{2}}$ : 


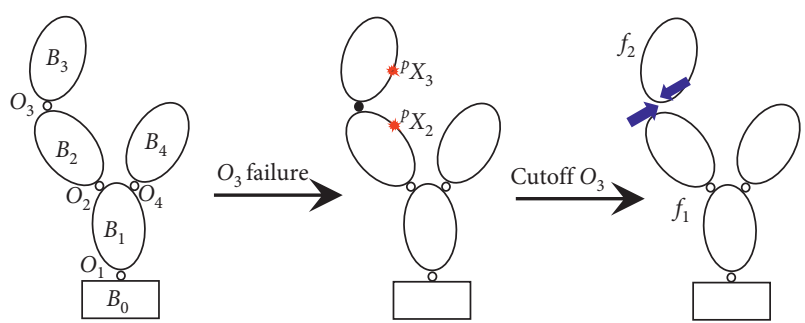

FIgURE 2: The mobile impact motion system.

$$
\begin{aligned}
& { }^{p} \dot{X}_{2}=p\left[\mathbf{J}_{q_{f_{1}}}^{v}\right]{ }^{p} \dot{\mathbf{q}}_{f_{1}}, \\
& { }^{p} \dot{X}_{3}=p\left[\mathbf{J}_{q_{f_{2}}}^{v}\right]{ }^{p} \dot{\mathbf{q}}_{f_{2}},
\end{aligned}
$$

where

$$
p\left[\mathbf{J}_{q_{f_{i}}}^{v}\right]=\frac{\partial\left({ }^{p} \boldsymbol{\varphi}_{f_{i}}\right)}{\partial\left({ }^{p} \mathbf{q}_{f_{i}}\right)^{\mathrm{T}}}, \quad(i=1,2) .
$$

When the system satisfies the conditions of configuration transformation, the mobile impact force ${ }^{p} F_{m_{i}}(i=1,2)$ of the multibody system $f_{i}$ is immediately generated. Based on the Newton-Euler equation, the multibody systems $f_{1}$ and $f_{2}$ with mobile impact motion are subject to the constraint forces at the cutoff joint $\mathrm{O}_{3}$ and the mobile impact forces in addition to driven and elastic forces. Therefore, the dynamic equations of the multibody systems $f_{1}$ and $f_{2}$ with mobile impact motion can be established as

$$
{ }^{p} \mathbf{D}_{f_{i}}{ }^{p} \ddot{\mathbf{q}}_{f_{i}}+{ }^{p} \mathbf{H}_{f_{i}}={ }^{p} \boldsymbol{\tau}_{a f_{i}}+{ }^{p} \boldsymbol{\tau}_{c f_{i}}+{ }^{p} \boldsymbol{\tau}_{\mathrm{cut} f_{i}}+{ }^{p} \boldsymbol{\tau}_{m f_{i}}, \quad(i=1,2),
$$

where ${ }^{p} \ddot{\mathbf{q}}_{f_{i}}$ is the generalized acceleration vector of the multibody system $f_{i},{ }^{p} \mathbf{D}_{f_{i}}$ is the inertia matrix of the multibody system $f_{i},{ }^{p} \mathbf{H}_{f_{i}}$ is the gravity, the Coriolis, and the centripetal force vector of the multibody system $f_{i},{ }^{p} \tau_{a f_{i}}$ is the generalized force vector of the multibody system $f_{i}$, ${ }^{p} \boldsymbol{\tau}_{c f_{i}}$ is the generalized elastic and other generalized force vector of the multibody system $f_{i},{ }^{p} \boldsymbol{\tau}_{\text {cut } f_{i}}$ is the generalized constraint force vector at the cutoff joint $\mathrm{O}_{3}$ of the multibody system $f_{i}$, and ${ }^{p} \boldsymbol{\tau}_{m f_{i}}$ is the generalized mobile impact force vector of the multibody system $f_{i}$.
According to the principle of virtual work, the generalized constraint force vector and the generalized mobile impact force vector of the multibody systems $f_{1}$ and $f_{2}$ at the cutoff joint $\mathrm{O}_{3}$ can be respectively obtained:

$$
\begin{gathered}
{ }^{p} \boldsymbol{\tau}_{\text {cut } f_{i}}=p\left[\mathbf{J}_{q_{f_{i}}}^{\mathrm{cut}}\right]^{\mathrm{T}}{ }^{p} F_{\text {cut } f_{i}}, \quad(i=1,2), \\
{ }^{p} \tau_{m f_{i}}=p\left[J_{q_{f_{i}}}^{v}\right]^{\mathrm{T}}{ }^{p} F_{m_{i}}, \quad(i=1,2),
\end{gathered}
$$

where ${ }^{p} \mathbf{J}_{q_{f_{i}}}^{\text {cut }}$ is the first-order kinematic influence coefficient of the velocity vector of the cutoff joint $\mathrm{O}_{3}$ in the inertial coordinate system with respect to the generalized velocity in the multibody system $f_{i},{ }^{p} F_{\text {cut } f_{i}}$ is the constraint force at the cutoff joint $\mathrm{O}_{3}$ of the multibody system $f_{i},{ }^{p} F_{\operatorname{cut} f_{i}}=-{ }^{p} F_{\text {cut } f_{2}}$, and ${ }^{P} F_{m_{1}}=-{ }^{P} F_{m_{2}}$.

According to the Newton-Euler equation, the constraint force at the cutoff joint $\mathrm{O}_{3}$ can be deduced in the form of generalized acceleration vector ${ }^{p} \ddot{\mathbf{q}}$ as

$$
{ }^{p} F_{\text {cut } f_{2}}={ }^{p} \mathbf{D}_{\text {cut }}{ }^{p} \ddot{\mathbf{q}}+{ }^{p} \mathbf{H}_{\text {cut }},
$$

where ${ }^{p} \ddot{\mathbf{q}}$ is the generalized acceleration vector of the mobile impact motion system, $P \mathbf{D}_{\text {cut }}$ is the coefficient matrix of ${ }^{p} F_{\text {cut } f_{2}}$ with respect to ${ }^{p} \ddot{\mathbf{q}}$, and ${ }^{p} \mathbf{H}_{\text {cut }}$ is a column matrix.

Combining the kinematic constraint equation at the cutoff joint $\mathrm{O}_{3}$, it can be obtained as

$$
{ }^{p} \ddot{\mathbf{q}}={ }^{p} \mathbf{A}^{p} \ddot{\mathbf{q}}_{f_{2}}+{ }^{p} \mathbf{B}
$$

where ${ }^{p} \mathbf{A}$ is the coefficient matrix of ${ }^{p} \ddot{\mathbf{q}}$ with respect to ${ }^{p} \ddot{\mathbf{q}}_{f_{2}}$ and ${ }^{P} \mathbf{B}$ is a column matrix.

Substituting equation (18) into equation (17), the constraint force ${ }^{p} F_{\text {cut } f_{2}}$ at the cutoff joint $\mathrm{O}_{3}$ can be derived as

$$
{ }^{p} F_{\text {cut } f_{2}}={ }^{p} \mathbf{D}_{\text {cut } f_{2}}{ }^{p} \ddot{\mathbf{q}}_{f_{2}}+{ }^{p} \mathbf{H}_{\text {cut } f_{2}},
$$

where ${ }^{p} \mathbf{D}_{\text {cut } f_{2}}={ }^{p} \mathbf{D}_{\text {cut }}{ }^{p} \mathbf{A}$ and ${ }^{p} \mathbf{H}_{\text {cut } f_{2}}={ }^{p} \mathbf{D}_{\text {cut }}{ }^{p} \mathbf{B}+{ }^{p} \mathbf{H}_{\text {cut }}$.

Combining equations (14)-(16) and (19), the mobile impact dynamic equation of PCMM can be derived as

$$
\left\{\begin{array}{l}
{ }^{p} \mathbf{D}_{f_{1}}{ }^{p} \ddot{\mathbf{q}}_{f_{1}}+{ }^{p} \mathbf{H}_{f_{1}}={ }^{p} \boldsymbol{\tau}_{a f_{1}}+{ }^{p} \boldsymbol{\tau}_{c f_{1}}-p\left[\mathbf{J}_{q_{f_{1}}}^{\mathrm{cut}}\right]^{\mathrm{T}}{ }^{p} \mathbf{D}_{\mathrm{cut} f_{2}}{ }^{p} \ddot{\mathbf{q}}_{f_{2}}{ }^{p}-\left[\mathbf{J}_{q_{f_{1}}}^{\mathrm{cut}}\right]^{\mathrm{T}}{ }^{p} \mathbf{H}_{\mathrm{cut} f_{2}}+p\left[\mathbf{J}_{q_{f_{1}}}^{v}\right]^{\mathrm{T}}{ }^{p} F_{m_{1}}, \\
{ }^{p} \mathbf{D}_{f_{2}} p \sum \ddot{\mathbf{q}}_{f_{2}}+{ }^{p} \mathbf{H}_{f_{2}}={ }^{p} \boldsymbol{\tau}_{a f_{2}}+{ }^{p} \boldsymbol{\tau}_{c f_{2}}+p\left[\mathbf{J}_{q_{f_{2}}}^{\mathrm{cut}}\right]^{\mathrm{T}}{ }^{p} \mathrm{D}_{\mathrm{cut} f_{2}}{ }^{p} \ddot{\mathbf{q}}_{f_{2}}+p\left[\mathbf{J}_{q_{f_{2}}}^{\mathrm{cut}}\right]^{\mathrm{T}}{ }^{p} \mathbf{H}_{\mathrm{cut} f_{2}}-p\left[\mathbf{J}_{q_{f_{2}}}^{v}\right]^{\mathrm{T}}{ }^{p} F_{m_{1}} .
\end{array}\right.
$$

It is emphasized that with respect to PCMM with closed-loop constraints, it can be transformed into multibody systems in the tree system by cutting off the metamorphic joints in the loop when PCMM is changed from one configuration to the next, so that its impact dynamic analysis can be carried out by the tree system theory mentioned above.

\section{The Impulse Solving Model of Constrained Metamorphic Mechanism}

Based on the dynamic equation in the sub-configuration movement stage, the system state variables before configuration transformation can be obtained. Aiming at the specific impact motion of mechanism, how to determine the 
internal impulse generated by configuration transformation instantaneously and the state variables of the system after configuration transformation is the basic research scope of the impact motion, and it is also an urgently solved core problem. In this paper, on the basis of the impact dynamic equations (10) and (20), the stable impulse solving model and the mobile impulse solving model can be, respectively, derived by combining the coefficient of restitution equation. The resulting system state variables after configuration transformation can be deduced according to variables before configuration transformation.

3.1. The Contact Impact Model. According to the classical collision theory, the contact-impact model has been developed with the following assumptions: (1) the impact time is infinitely small; (2) the position and orientation of all components remain unchanged during the process of configuration transformation; (3) the impact is a point contact; (4) the shape and the inertia of the component are unchanged during the process of configuration transformation.

In general, the impact is partially elastic, and $e(0<e<1)$ is the coefficient of restitution. The normal vector of the impact point velocity increment is $n$ along the contact surface, and the stable and mobile impact motion systems should satisfy the coefficient of restitution equation at the instant of configuration transformation as follows:

$$
\left(\Delta v_{1}-\Delta v_{2}\right)^{\mathrm{T}} n=-(1+e)\left(v_{1}-v_{2}\right)^{\mathrm{T}} n
$$

where $v_{1}$ and $v_{2}$ are the velocities of impact points relative to the inertial coordinate system before configuration transformation and $\Delta v_{1}$ and $\Delta v_{2}$ are the velocity increments immediately after configuration transformation.

3.2. The Stable Impulse Solving Model. According to the basic assumption, the generalized positions of the system remain unchanged over a short duration $t_{0}<t<t_{0}+\Delta t$ where the time interval $\Delta t$ is small enough, so its generalized velocities are limited. The derived stable impact dynamic equation (10) is integrated over the time interval $\Delta t$, and the result is obtained as

$$
\begin{aligned}
& \int_{t_{0}}^{t_{0}+\Delta t}\left({ }^{p} \mathbf{D}_{f}{ }^{p} \ddot{\mathbf{q}}_{f}-p\left[\mathbf{J}_{q_{f}}^{\text {cut }}\right]^{\mathrm{T}}{ }^{p} \mathbf{D}_{\text {cut } f}{ }^{p} \ddot{\mathbf{q}}_{f}\right) \mathrm{d} t+\int_{t_{0}}^{t_{0}+\Delta t}{ }^{p} \mathbf{H}_{f} \mathrm{~d} t \\
& =\int_{t_{0}}^{t_{0}+\Delta t}\left({ }^{p} \boldsymbol{\tau}_{a f}+{ }^{p} \boldsymbol{\tau}_{c f}+{ }^{p}\left[\mathbf{J}_{q_{f}}^{\mathrm{cut}}\right]^{\mathrm{T}}{ }^{p} \mathrm{H}_{\text {cut } f}\right) \mathrm{d} t \\
& \quad+\int_{t_{0}}^{t_{0}+\Delta t} p\left[\mathbf{J}_{q_{f}}^{v}\right]^{\mathrm{T}}{ }^{p} F_{s} \mathrm{~d} t .
\end{aligned}
$$

Taking the limit of equation (22) as $\Delta t$ tends to zero, it can be written as

$$
\begin{aligned}
& \left({ }^{p} \mathbf{D}_{f}-p\left[\mathbf{J}_{q_{f}}^{\mathrm{cut}}\right]^{\mathrm{T}}{ }^{p} \mathbf{D}_{\text {cut } f}\right)\left({ }^{p} \dot{\mathbf{q}}_{f}\left(t_{0}+\Delta t\right)-{ }^{p} \dot{\mathbf{q}}_{f}\left(t_{0}\right)\right) \\
& ={ }^{p}\left[\mathbf{J}_{q_{f}}^{v}\right]^{\mathrm{T}}{ }^{p} \mathbf{P}_{s},
\end{aligned}
$$

where $P \mathbf{P}_{s}=\int_{t_{0}}^{t_{0}+\Delta t} p F_{s} \mathrm{~d} t$ is defined as the stable impulse at the impact point.

Then, the velocity increments of the generalized positions can be expressed as

$$
\Delta^{p} \dot{\mathbf{q}}_{f}=\left({ }^{p} \mathbf{M}_{f}\right)^{-1} p\left[\mathbf{J}_{q_{f}}^{v}\right]^{\mathrm{T}}{ }^{p} P_{s}
$$

where

$$
{ }^{p} \mathbf{M}_{f}={ }^{p} \mathbf{D}_{f}-p\left[\mathbf{J}_{q_{f}}^{\text {cut }}\right]^{\mathrm{T}}{ }^{p} \mathbf{D}_{\text {cut } f}
$$

Based on the kinematics, the velocity increment of the impact point can be written as

$$
\Delta v_{1}={ }^{p}\left[{ }^{p} \mathbf{J}_{q_{f}}\right]\left({ }^{p} \mathbf{M}_{f}\right)^{-1} p\left[\mathbf{J}_{q_{f}}^{v}\right]^{\mathrm{T}}{ }^{p} P_{s}
$$

According to the stable impact motion between the moving component and the frame, the absolute velocity and the velocity increment of the frame are always zero $\left(v_{2}=\Delta v_{2}=0\right)$. Assuming that there is no friction between two impacting surfaces and substituting equation (26) into equation (21), it can be obtained as

$$
\left(p\left[\mathbf{J}_{q_{f}}^{v}\right]\left({ }^{p} \mathbf{M}_{f}\right)^{-1} p\left[\mathbf{J}_{q_{f}}^{v}\right]^{\mathrm{T}}{ }^{p} P_{s}\right)^{\mathrm{T}} n=-(1+e) v_{1}^{\mathrm{T}} n .
$$

In addition,

$$
{ }^{p} P_{s}={ }^{p} P_{s} n
$$

Then, the stable impulse of PCMM can be expressed as

$$
{ }^{p} P_{s}=\frac{-(1+e) v_{1}^{\mathrm{T}} n}{\mathbf{n}^{T}\left(p\left[\mathbf{J}_{q_{f}}^{v}\right]\left(p \mathbf{M}_{f}\right)^{-1} p\left[\mathbf{J}_{q_{f}}^{v}\right]^{\mathrm{T}}\right)^{\mathrm{T}} \mathbf{n}} .
$$

Combining the initial condition of the system before configuration transformation, the generalized velocity of the system after configuration transformation can be obtained as

$$
{ }^{p} \dot{\mathbf{q}}_{f}^{+}=\Delta^{p} \dot{\mathbf{q}}_{f}+{ }^{p} \dot{\mathbf{q}}_{f}^{-},
$$

where ${ }^{P} \dot{\mathbf{q}}_{f}^{+}$is the generalized velocity vector after configuration transformation and ${ }^{P} \dot{\mathbf{q}}_{f}^{-}$is the generalized velocity vector before configuration transformation.

3.3. The Mobile Impulse Solving Model. According to the basic assumption, the derived mobile impact dynamic equation (20) is integrated over the time interval $\Delta t$, and the result is obtained as 


$$
\begin{aligned}
& \int_{t_{0}}^{t_{0}+\Delta t}{ }^{p} \mathbf{D}_{f_{1}}{ }^{p} \ddot{\mathbf{q}}_{f_{1}} \mathrm{~d} t+\int_{t_{0}}^{t_{0}+\Delta t} p\left[\mathbf{J}_{q_{f_{1}}}^{\text {cut }}\right]^{\mathrm{T}}{ }^{p} \mathbf{D}_{\text {cut } f_{2}}{ }^{p} \ddot{\mathbf{q}}_{f_{2}} \mathrm{~d} t+\int_{t_{0}}^{t_{0}+\Delta t}{ }^{p} \mathbf{H}_{f_{1}} \mathrm{~d} t \\
& =\int_{t_{0}}^{t_{0}+\Delta t}\left({ }^{p} \boldsymbol{\tau}_{a f_{1}}+{ }^{p} \boldsymbol{\tau}_{c f_{1}}-p\left[\mathbf{J}_{q_{f_{1}}}^{\text {cut }}\right]^{\mathrm{T}}{ }^{\mathrm{T}} \mathbf{H}_{\mathrm{cut} f_{2}}\right) \mathrm{d} t \\
& +\int_{t_{0}}^{t_{0}+\Delta t} p\left[\mathbf{J}_{q_{f_{1}}}^{v}\right]^{\mathrm{T}}{ }^{\mathrm{T}} \mathbf{F}_{m_{1}} \mathrm{~d} t \\
& \int_{t_{0}}^{t_{0}+\Delta t}\left({ }^{p} \mathbf{D}_{f_{2}}{ }^{p} \ddot{\mathbf{q}}_{f_{2}}-{ }^{p}\left[\mathbf{J}_{q_{f_{2}}}^{\text {cut }}\right]^{\mathrm{T}}{ }^{p} \mathbf{D}_{\operatorname{cut} f_{2}}{ }^{p} \ddot{\mathbf{q}}_{f_{2}}\right) \mathrm{d} t+\int_{t_{0}}^{t_{0}+\Delta t}{ }^{p} \mathbf{H}_{f_{2}} \mathrm{~d} t \\
& =\int_{t_{0}}^{t_{0}+\Delta t}\left({ }^{p} \boldsymbol{\tau}_{a f_{2}}+{ }^{p} \boldsymbol{\tau}_{c f_{2}}+{ }^{p}\left[\mathbf{J}_{q_{f_{2}}}^{\text {cut }}\right]^{\mathrm{T}}{ }^{p} \mathbf{H}_{\mathrm{cut} f_{2}}\right) \mathrm{d} t \\
& -\int_{t_{0}}^{t_{0}+\Delta t} p\left[\mathbf{J}_{q_{f_{2}}}^{v}\right]^{\mathrm{T}}{ }^{p} \mathbf{F}_{m_{1}} \mathrm{~d} t \text {. }
\end{aligned}
$$

Since the mobile impact motion is of short duration and generates a large mutual impact force, the generalized positions of PCMM are not significantly changed during configuration transformation and only the generalized velocities are changed. Based on the above analysis, equations (31) and (32) can be approximately written as

$$
\begin{aligned}
& p\left[\mathbf{J}_{q_{f_{1}}}^{\text {cut }}\right]^{\mathrm{T}}{ }^{p} \mathbf{D}_{\text {cut } f_{2}}\left({ }^{p} \dot{\mathbf{q}}_{f_{2}}\left(t_{0}+\Delta t\right)-{ }^{p} \dot{\mathbf{q}}_{f_{2}}\left(t_{0}\right)\right) \\
& +{ }^{p} \mathbf{D}_{f_{1}}\left({ }^{p} \dot{\mathbf{q}}_{f_{1}}\left(t_{0}+\Delta t\right)-{ }^{p} \dot{\mathbf{q}}_{f_{1}}\left(t_{0}\right)\right)={ }^{p}\left[\mathbf{J}_{q_{f_{1}}}^{v}\right]^{\mathrm{T}}{ }^{p} P_{m_{1}}, \\
& \left({ }^{p} \mathbf{D}_{f_{2}}-{ }^{p}\left[\mathbf{J}_{q_{f_{2}}}^{\mathrm{cut}}\right]^{\mathrm{T}}{ }^{p} \mathbf{D}_{\mathrm{cut} f_{2}}\right)\left({ }^{p} \dot{\mathbf{q}}_{f_{2}}\left(t_{0}+\Delta t\right)-{ }^{p} \dot{\mathbf{q}}_{f_{2}}\left(t_{0}\right)\right) \\
& =--^{p}\left[\mathbf{J}_{q_{f_{2}}}^{v}\right]^{\mathrm{T}}{ }^{\mathrm{T}} P_{m_{1}}
\end{aligned}
$$

where ${ }^{p} \mathbf{P}_{m_{1}}=\int_{t_{0}}^{t_{0}+\Delta t}{ }^{p} \mathbf{F}_{m_{1}} \mathrm{~d} t$ is defined as the mobile impulse at the impact point.

The derivation of (33) and (34) is based on the conservation of system momentum. Based on equation (34), the velocity increments of the generalized positions can be expressed in the multibody system $f_{2}$ as

$$
\Delta^{p} \dot{q}_{f_{2}}=-\left({ }^{p} \mathbf{M}_{f_{2}}\right)^{-1} p\left[\mathbf{J}_{\mathbf{q}_{f_{2}}^{v}}\right]^{\mathrm{T}}{ }^{p} P_{m_{1}}
$$

where

$$
{ }^{p} \mathbf{M}_{f_{2}}={ }^{p} \mathbf{D}_{f_{2}}-{ }^{p}\left[\mathbf{J}_{\mathbf{q}_{f_{2}}}^{\text {cut }}\right]^{\mathrm{T}}{ }^{p} \mathbf{D}_{\text {cut } f_{2}} \cdot
$$

Based on the kinematics, the velocity increment of the impact point in the multibody system $f_{2}$ can be written as

$$
\Delta v_{2}=-{ }^{p} \mathbf{J}_{\mathbf{q}_{f_{2}}}^{v}\left({ }^{p} \mathbf{M}_{f_{2}}\right)^{-1} p\left[\mathbf{J}_{\mathbf{q}_{f_{2}}}^{v}\right]^{\mathrm{T}}{ }^{p} P_{m_{1}} .
$$

Substituting equation (35) into equation (33), the velocity increment of the generalized coordinate in the multibody system $f_{1}$ can be expressed as

$$
\Delta^{p} \dot{q}_{f_{1}}={ }^{p} \mathbf{M}_{f_{1}}^{p} P_{m_{1}}
$$

where

$$
{ }^{p} \mathbf{M}_{f_{1}}={ }^{p} \mathbf{D}_{f_{1}}^{-1 p}\left[\mathbf{J}_{\mathbf{q}_{f_{1}}}^{\text {cut }}\right]^{\mathrm{T} p} \mathbf{D}_{\operatorname{cut} f_{2}}^{p}\left({ }^{p} \mathbf{M}_{f_{2}}\right)^{-1 p} p\left[\mathbf{J}_{\mathbf{q}_{f_{2}}}^{v}\right]^{\mathrm{T}}+{ }^{p} \mathbf{D}_{f_{1}}^{-1 p}\left[\mathbf{J}_{\mathbf{q}_{f_{1}}}^{\text {cut }}\right]^{\mathrm{T}} .
$$

Based on the kinematics, the velocity increment of the impact point in the multibody system $f_{1}$ can be written as

$$
\Delta v_{1}=p\left[\mathbf{J}_{q_{f_{1}}}^{v}\right] p \mathbf{M}_{f 1}^{p} P_{m_{1}}
$$

In addition,

$$
{ }^{p} P_{m_{1}}={ }^{p} P_{m_{1}} n .
$$

Substituting equations (37), (40), and (41) into (21), the mobile impulse of PCMM can be derived as

$$
{ }^{p} P_{m_{1}}=\frac{-(1+e)\left(v_{1}-v_{2}\right)^{\mathrm{T}} \mathbf{n}}{n^{\mathrm{T}}\left(p\left[\mathbf{J}_{q_{f_{1}}}^{v}\right]{ }^{p} \mathbf{M}_{f_{1}}+K\right)^{\mathrm{T}} \mathbf{n}},
$$

where $K=p\left[\mathbf{J}_{q_{f_{2}}}^{v}\right]\left(P_{\mathbf{M}_{2}}\right)^{-1} p\left[\mathbf{J}_{q_{f_{2}}}^{v}\right]^{T}$.

Combining the initial condition of the system before configuration transformation, the generalized velocity of the system after configuration transformation can be obtained as

$$
{ }^{p} \dot{\mathbf{q}}_{f_{i}}^{+}=\Delta^{p} \dot{\mathbf{q}}_{f_{i}}+{ }^{p} \dot{\mathbf{q}}_{f_{i}}^{-}, \quad i=1,2,
$$

where ${ }^{p} \dot{\mathbf{q}}_{f_{i}}^{+}$is the generalized velocity vector of the multibody system $f_{i}$ after configuration transformation and ${ }^{P} \dot{\mathbf{q}}_{f_{i}}^{-}$ is the generalized velocity vector of the multibody system $f_{i}$ before configuration transformation.

According to the above derivation results, if the impulse of the impact point in the system and the velocity of each component before configuration transformation are known, we can calculate the velocity of each component after configuration transformation.

\section{Dynamic Numerical Examples}

For the sake of completeness, this section lists two typical examples to model and simulate the impact dynamics. One is an open-loop constrained metamorphic mechanism and the other is a closed-loop constrained metamorphic mechanism. To analyze the motion characteristics of the system before and after configuration transformation, the dynamic simulations of PCMM can be carried out by MATLAB. Since the impact dynamic equation of the system is nonlinear, it can be solved by using Runge-Kutta method to realize the configuration-complete dynamic simulation of PCMM. The flow graph of numerical simulation is shown in Figure 3.

4.1. Simulation Example 1. In this case, the impact dynamics of the open-loop three-bar constrained metamorphic 


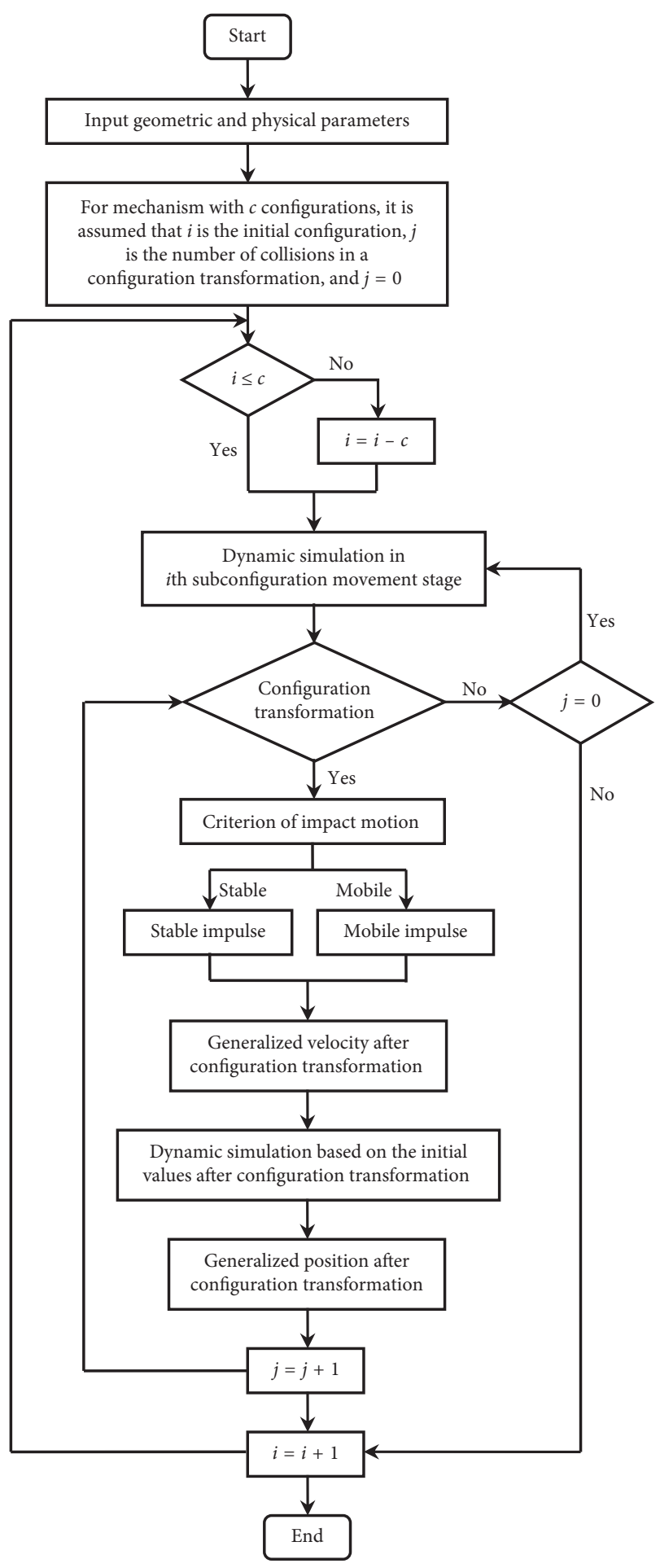

Figure 3: The flow graph of numerical simulation.

mechanism is analyzed in Figure 4. Symbols described in Figure 4 are explained as follows:

(1) $i(i=1,2,3)$ : component $i$ of the open-loop threebar constrained metamorphic mechanism

(2) $O_{i}(i=1,2,3)$ : joint $O_{i}$ of the open-loop three-bar constrained metamorphic mechanism
(3) $\theta_{i}(i=1,2,3)$ : the angle between component $i$ and the $x$-axis

(4) $\tau_{i}(i=1,2,3)$ : the equivalent moments acting on joint $O_{i}$

As shown in Figure 4, the open-loop three-bar constrained metamorphic mechanism is composed of component 1 , component 2, component 3, and the frame. Assume that the motor is installed at the joint $O_{1}$ and the torsion spring is installed at the joint $\mathrm{O}_{2}$. If $\theta_{3}-\theta_{2}<\pi(\mathrm{rad})$, the mechanism is in configuration 1 as shown in Figure 4(a); if $\theta_{3}-\theta_{2}=\pi(\mathrm{rad})$, the component 2 merges with the component 3 , and the mechanism is in configuration 2 as shown in Figure 4(b).

For the abovementioned metamorphic mechanism, which realizes the whole process of metamorphosis by constraints, it is required that the corresponding metamorphic joint of the corresponding configuration should be able to provide corresponding constraints in order to realize the configuration transformation in the working process. According to the existing working configuration, the joint $\mathrm{O}_{3}$ is chosen as the metamorphic joint and its main realization form is shown in Figure 5. Symbols described in Figure 5 are explained as follows:

\section{$a$ : a limited pin distributed on a circle}

Figure 5 shows a limited rotation metamorphic joint [37]. The structure of the metamorphic joint is obtained by synthesizing the pin-restrained rotation metamorphic joint and the spring-restrained rotation metamorphic joint. The component 2 and the component 3 are connected by torsion spring, and the component 2 contains a limited pin $a$ distributed on a circle.

Then, the equivalent moments acting on each joint can be expressed as follows, and the geometric and physical parameters of the open-loop three-bar constrained metamorphic mechanism are shown in Table 1.

$$
\begin{aligned}
\tau_{1} & =1.9550-0.0196 \dot{\theta}_{1}(\mathrm{~N} \cdot \mathrm{m}), \\
\tau_{2} & =0.1625-0.0161\left(\theta_{2}-\theta_{1}\right)(\mathrm{N} \cdot \mathrm{m}), \\
\tau_{3} & =0.2300-0.026\left(\theta_{3}-\theta_{2}\right)(\mathrm{N} \cdot \mathrm{m}) .
\end{aligned}
$$

Assuming that the open-loop three-bar constrained metamorphic mechanism is transformed from configuration 1 to configuration $2\left(\theta_{3}-\theta_{2}=\pi, t=t_{p}=0.1674 \mathrm{~s}\right)$, the centroid point of component 3 is impacted with the centroid point of component 2 . The normal vector of the impact point velocity increment is $\mathbf{n}=\left[\begin{array}{lll}\sin \theta_{2} & -\cos \theta_{2} & 0\end{array}\right]^{T}$ and the coefficient of restitution $e$ is 0.8 . Based on the dynamics, the absolute angular velocity of each component before configuration transformation can be calculated as follows:

$$
\left\{\begin{array}{l}
\omega_{10}^{-}=\dot{\theta}_{10}^{-}=2.8917(\mathrm{rad} / \mathrm{s}), \\
\omega_{20}^{-}=\dot{\theta}_{20}^{-}=-1.1867(\mathrm{rad} / \mathrm{s}), \\
\omega_{30}^{-}=\dot{\theta}_{30}^{-}=22.1639(\mathrm{rad} / \mathrm{s}) .
\end{array}\right.
$$

According to the mobile impulse solving model, the mobile impulse $P_{m_{1}}$ in the instantaneous configuration transformation can be obtained as 


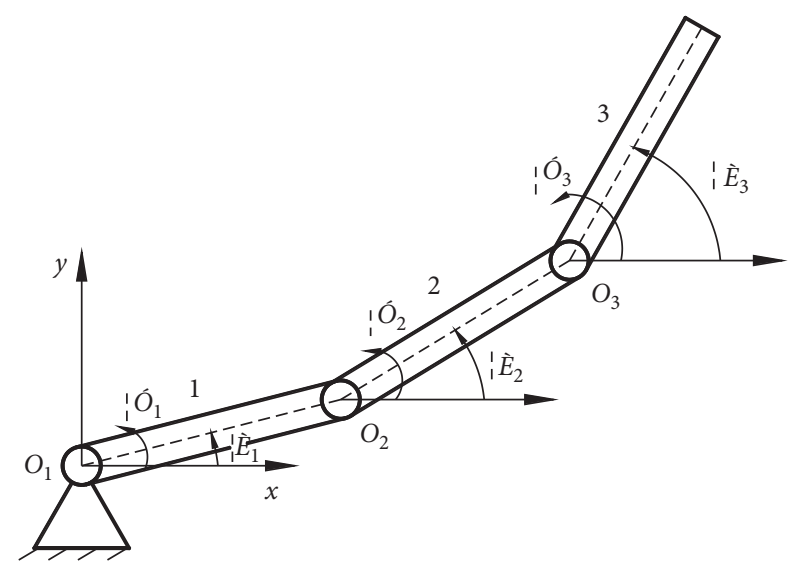

(a)

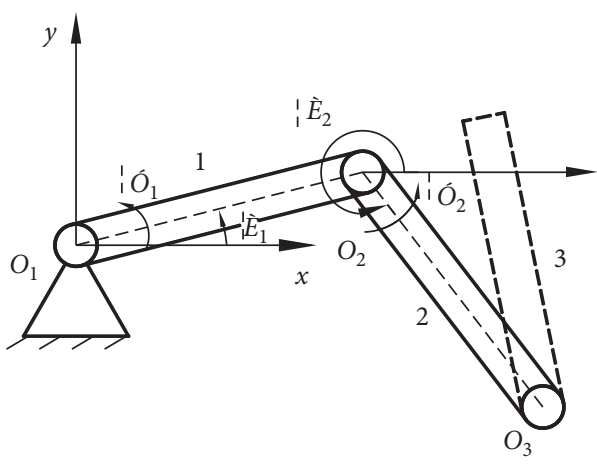

(b)

Figure 4: The open-loop three-bar constrained metamorphic mechanism. (a) Configuration 1. (b) Configuration 2.

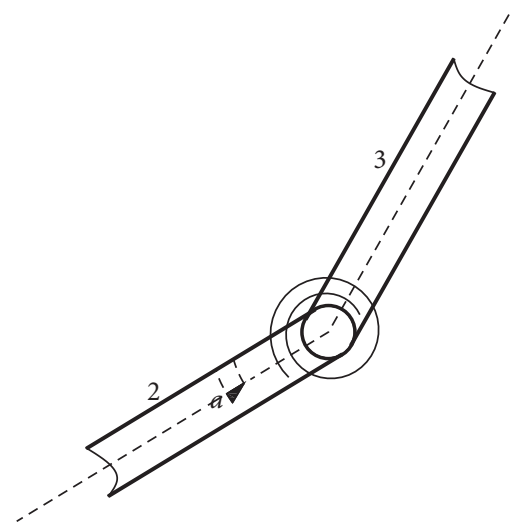

Figure 5: The limited rotation metamorphic joint.

TABLE 1: Geometric and physical parameters of the open-loop three-bar constrained metamorphic mechanism.

\begin{tabular}{lccc}
\hline & Link 1 & Link 2 & Link 3 \\
\hline Length $(\mathrm{m})$ & 0.25 & 0.125 & 0.125 \\
Cross-sectional area $\left(\mathrm{m}^{2}\right)$ & $2 \times 10^{-4}$ & $2 \times 10^{-4}$ & $2 \times 10^{-4}$ \\
Density $\left(\mathrm{kg} / \mathrm{m}^{3}\right)$ & 7800 & 7800 & 7800 \\
Initial angle $(\mathrm{rad})$ & 0 & 0 & 0 \\
Initial angular velocity $(\mathrm{rad} / \mathrm{s})$ & 0 & 0 & 0 \\
\hline
\end{tabular}

$$
P_{m_{1}}=\left[\begin{array}{lll}
-1.0070 & -0.1224 & 0
\end{array}\right]^{T}(\mathrm{~N} \cdot \mathrm{s}) .
$$

Based on equation (43), the absolute angular velocity of each component after configuration transformation can be calculated as follows:

$$
\left\{\begin{array}{l}
\omega_{10}^{+}=\dot{\theta}_{10}^{+}=4.5188(\mathrm{rad} / \mathrm{s}), \\
\omega_{20}^{+}=\dot{\theta}_{20}^{+}=0.0489(\mathrm{rad} / \mathrm{s}), \\
\omega_{30}^{+}=\dot{\theta}_{30}^{+}=-102.6927(\mathrm{rad} / \mathrm{s}) .
\end{array}\right.
$$

In addition, constrained metamorphic operations of the mechanism are implemented by using the geometric constraint and the force constraint of the metamorphic joint $\mathrm{O}_{3}$ to make it locked. Owing to the existence of the geometric constraint, the metamorphic joint $\mathrm{O}_{3}$ is locked in instantaneous configuration transformation. Therefore, component 2 is merged with component 3 after configuration transformation, that is, $\dot{\theta}_{30}^{+}=\dot{\theta}_{20}^{+}$, when considering constraint conditions of configuration transformation.

The numerical simulation results of each component are shown in Figures 6-9. In the conditions of considering and ignoring the impact motion during configuration transformation, the comparisons of the absolute angle of each component in the open-loop three-bar constrained metamorphic mechanism are shown in Figures 6(a)-6(c), respectively. Similarly, Figure $6(\mathrm{~d})$ shows the angle of component 3 relative to component 2 .

From Figures 6(a)-6(c), it can be seen that the motion trends of components considering the impact motion are 


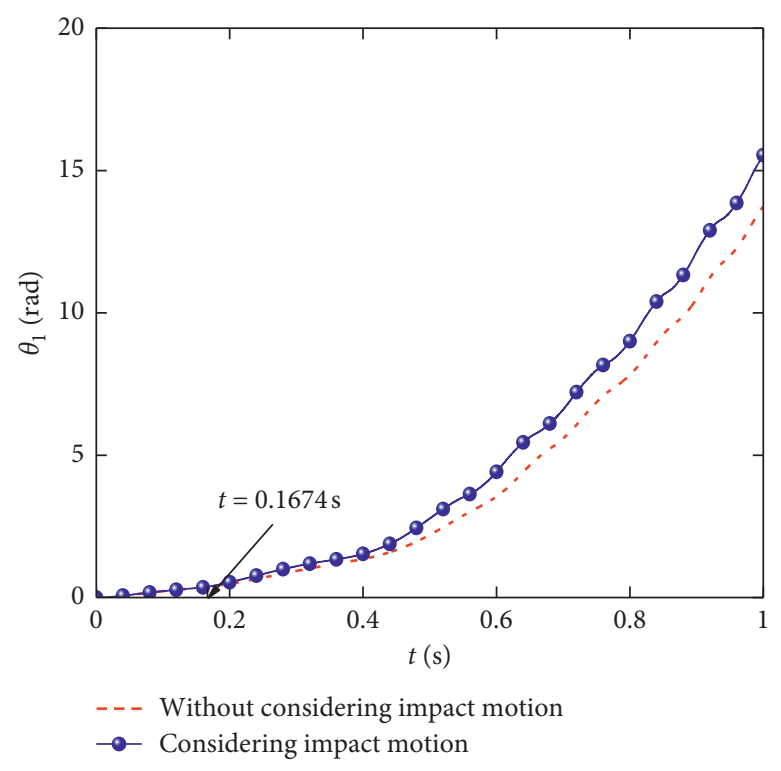

(a)

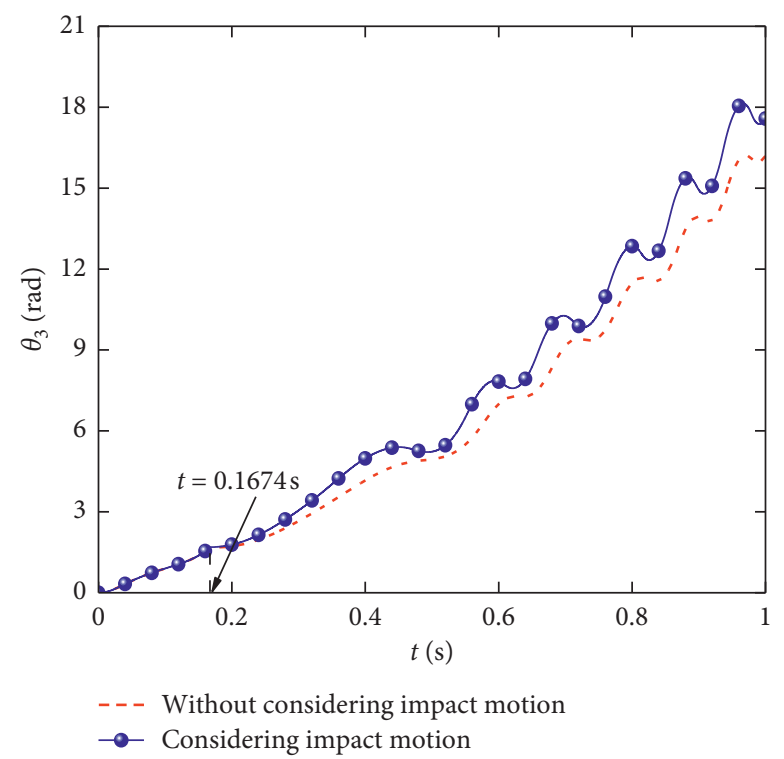

(c)

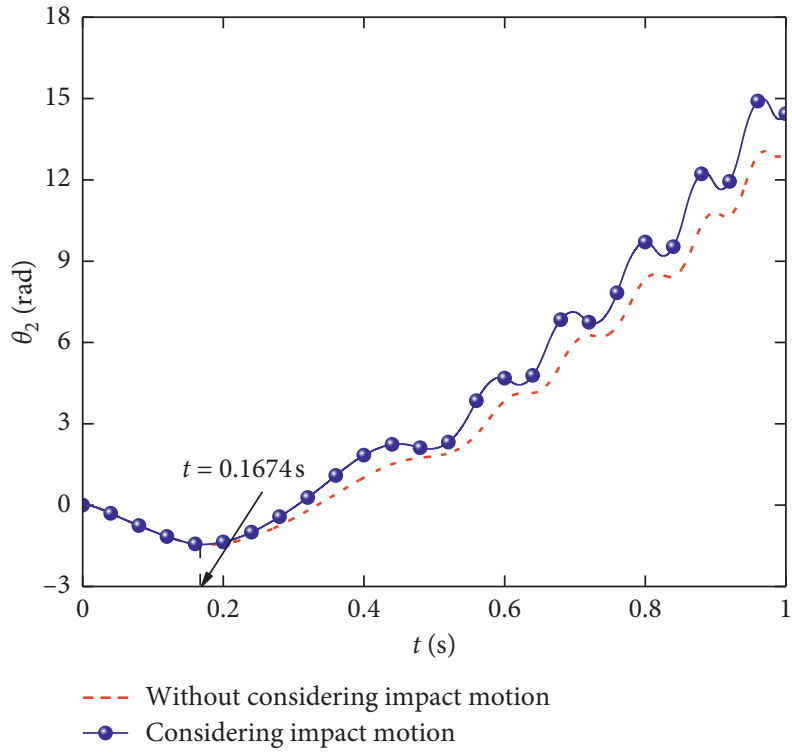

(b)

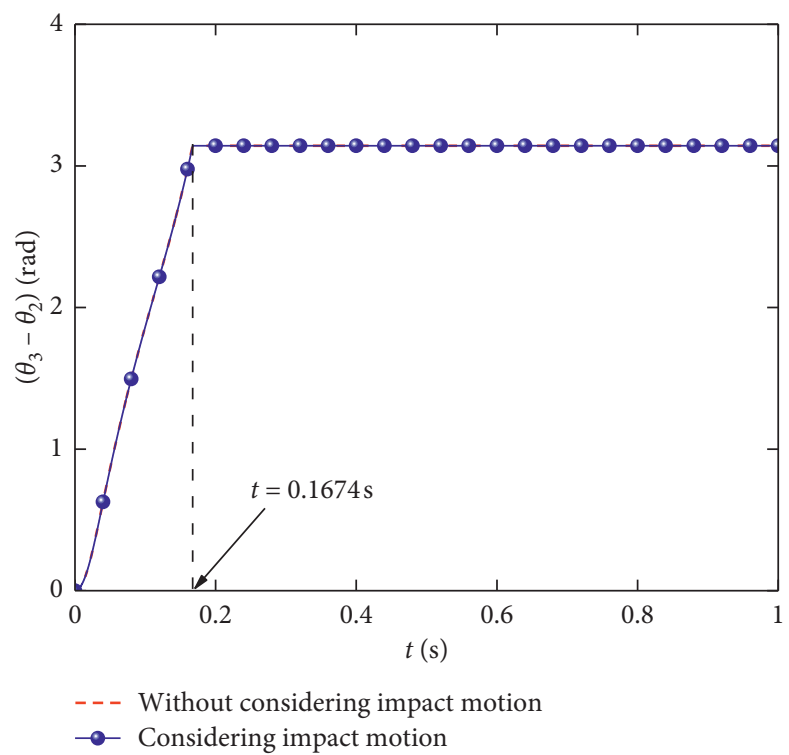

(d)

Figure 6: Angular displacement simulations. (a) Variation in the angle of component 1. (b) Variation in the angle of component 2. (c) Variation in the angle of component 3. (d) Angle variation of component 3 relative to 2.

exactly different from the motion trends ignoring the impact motion. Moreover, the motion amplitudes of each component considering the impact motion are larger than that of each component ignoring the impact motion, which indicates that the impact motion has a positive influence on each component. The motion law of component 3 relative to component 2 does not encounter changes, as shown in Figure $6(\mathrm{~d})$, which is determined by the conditions of configuration transformation.

The comparisons of the absolute angular velocities are shown in Figures $7(\mathrm{a})-7(\mathrm{c})$, respectively. The absolute angular velocity of each component after configuration transformation has an obvious abrupt change, as shown in Figure 7, and it illustrates that the impact will occur in each joint with considering the impact motion during configuration transformation. In addition, both component 2 and component 3 are subjected to the mobile impact, and the abrupt change of absolute angular velocity of component 3 is obviously higher than component 2 with the same structural parameters, which is determined by the state variables of the system before configuration transformation.

Figure 8 compares and analyses the moments acting on the joints in the conditions of considering and ignoring the impact motion during configuration transformation. The moment at joint $O_{1}$ is related to the angular velocity of the 


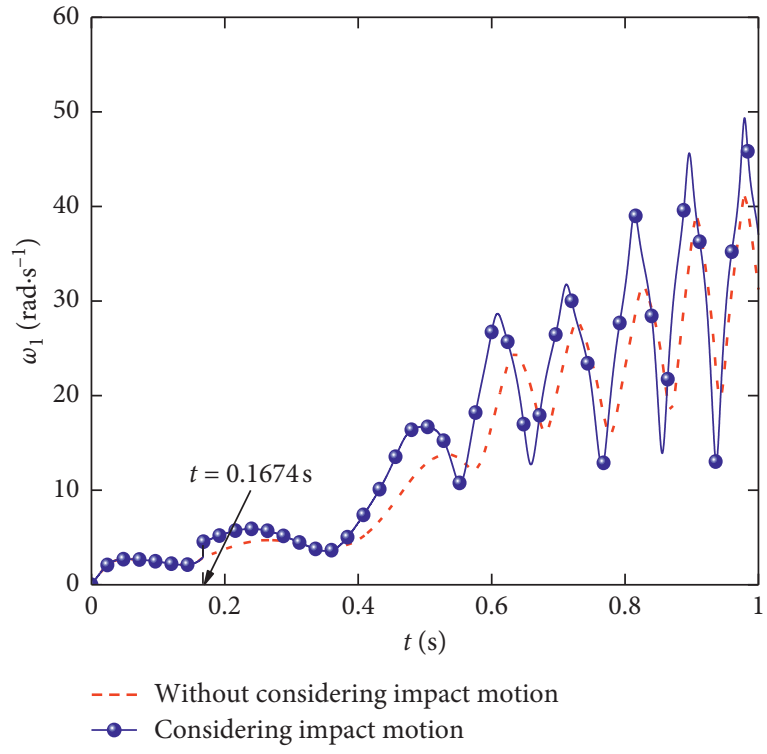

(a)

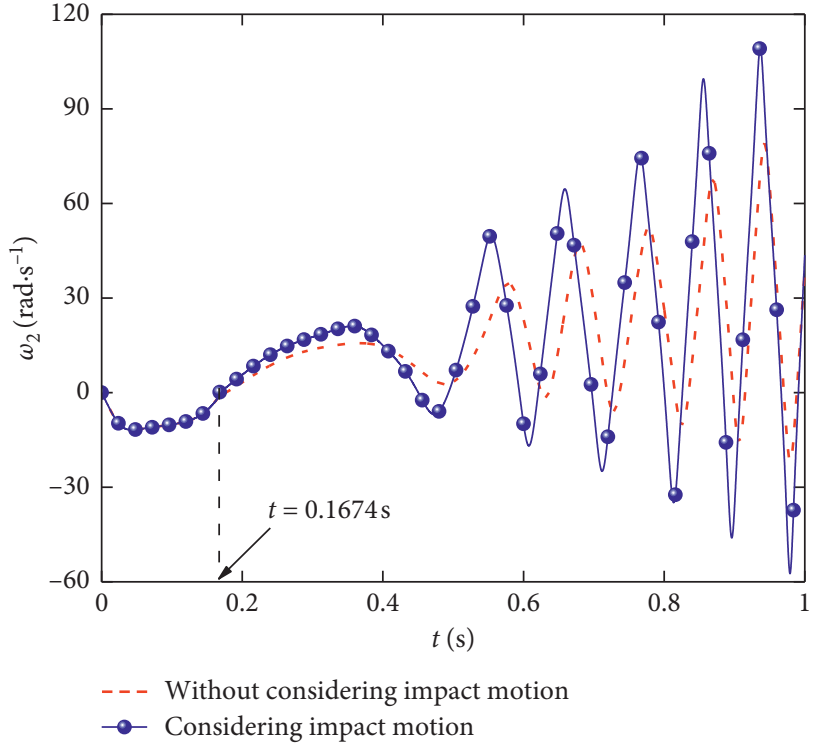

(b)

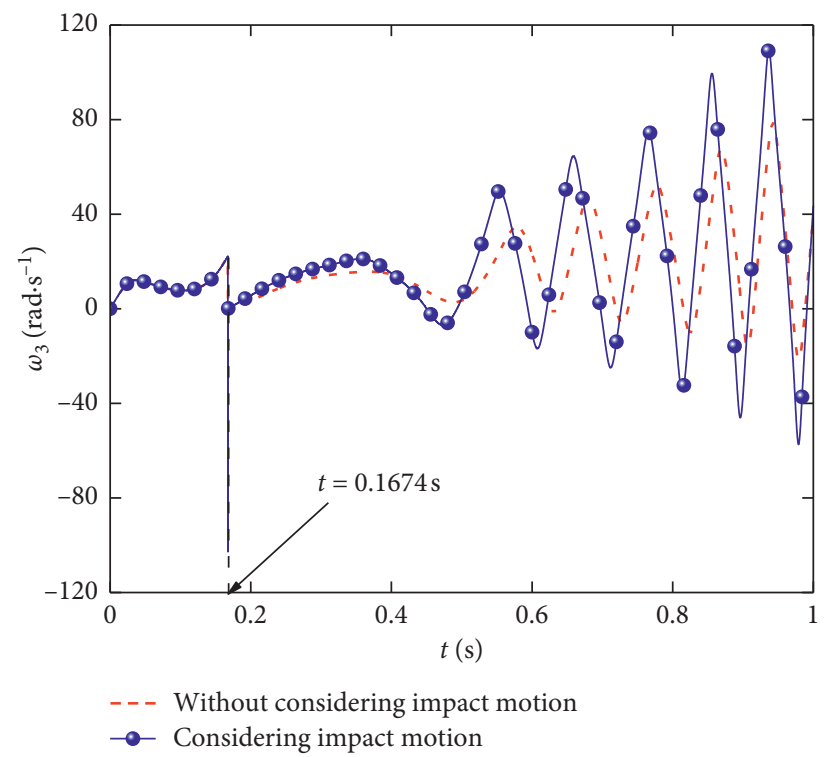

(c)

Figure 7: Angular velocity simulations. (a) Angular velocity variation of component 1. (b) Angular velocity variation of component 2. (c) Angular velocity variation of component 3 .

component 1 , so the moment changes abruptly after configuration transformation. The moments at joints $\mathrm{O}_{2}$ and $\mathrm{O}_{3}$ are only related to their absolute angular displacements, and there is no abrupt change in the whole movement process.

Figure 9 shows the variation of dynamic behavior for the system in the whole simulation process from the perspective of energy. As shown in Figure 9, kinematic energy of the system changes abruptly at the instant of configuration transformation. This is due to the impact of configuration transformation. Since potential energy is related to the absolute angular displacement, there is no abrupt change at the instant of configuration transformation.
4.2. Simulation Example 2. Taking the planar double-folded metamorphic mechanism as an example [38], the types and constraints of metamorphic joints based on the kinematic characteristics of metamorphic joints are given. According to the working task, the planar double-folded metamorphic mechanism (as shown in Figure 10) must complete the following two working requirements: horizontal folding and vertical folding:

(1) Horizontal folding, as shown in Figure 10(a): horizontally pushing the left side of the single-layer cardboard and rotating the second side of the left side along the second crease to the vertical position 


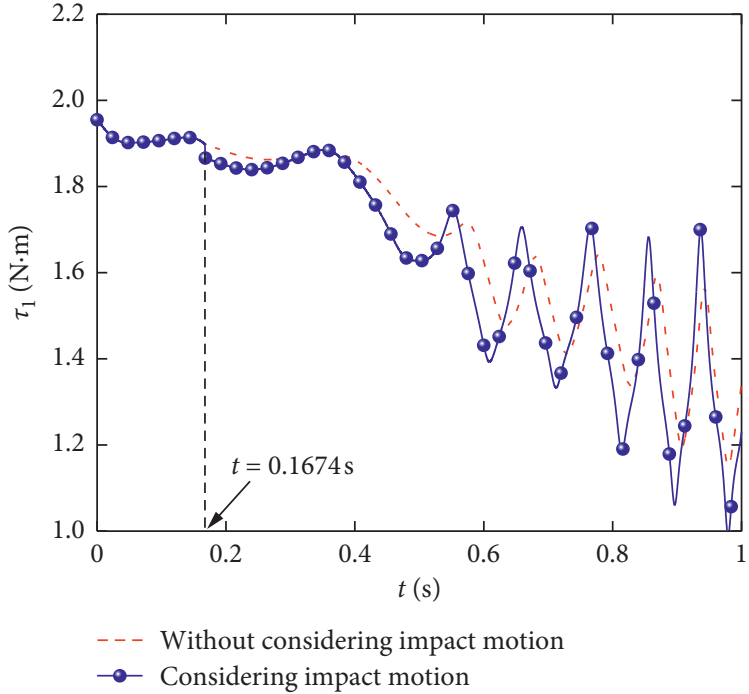

(a)

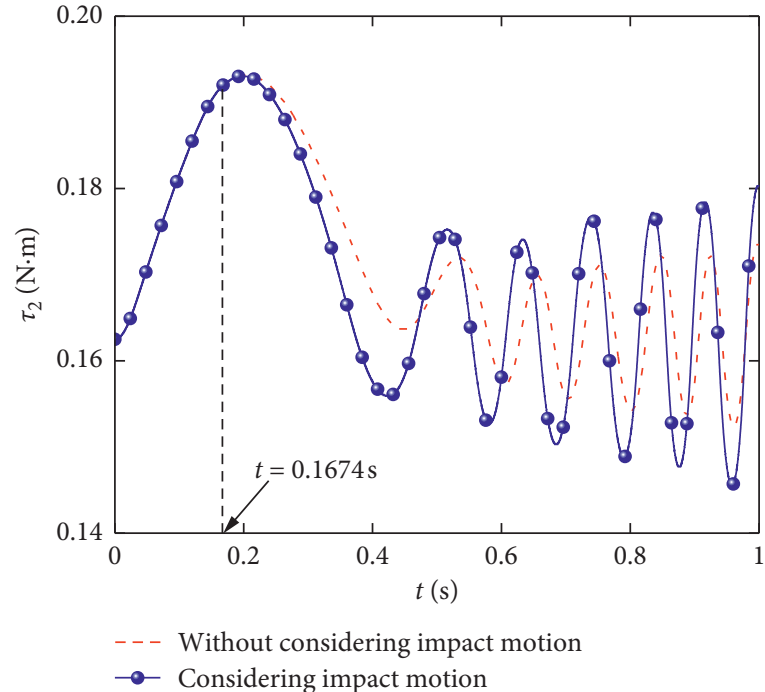

(b)

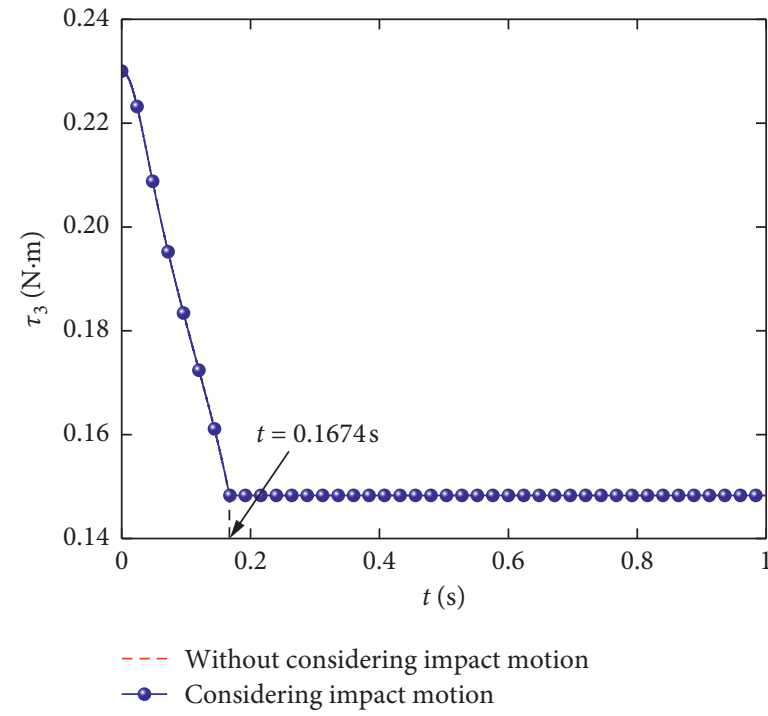

(c)

Figure 8: Moment simulations of the joints. (a) Moment variation of joint $O_{1}$. (b) Moment variation of joint $\mathrm{O}_{2}$. (c) Moment variation of joint $\mathrm{O}_{3}$.

(2) Vertical folding, as shown in Figure 10(b): completing the rotation of the first surface around the first crease and coinciding with the second surface

Figure 11 shows the schematic diagram of the planar double-folded metamorphic mechanism. According to the above requirements, force metamorphism is adopted at joint $D$, that is, when the mechanism is in configuration 1 , the spring force is set at joint $D$, so that the relative moving resistance between the components 3 and 4 is larger than the motion resistance of the slider, and it keeps the components 3 and 4 relatively static. When the mechanism is in configuration 2, a geometric constraint is added at joint $E$, so that the slider moves to the specified position and stops moving when the mechanism meets the geometric limit. Its geometric and physical parameters are shown in Table 2.
Assume that the motion law of component 1 can be written as follows:

$$
\theta_{1}=\pi+\frac{\pi}{5} t(\mathrm{rad}) .
$$

In view of the abovementioned conditions, the numerical simulation is carried out, as shown in Figures 12-15. Figure 12 shows the change of impulse when the planar double-folded metamorphic mechanism is transformed from configuration 1 to configuration 2 .

From Figure 12, it can be seen that there is a macroimpact behavior in the whole simulation process. Since the impact is partially elastic and the impact point on the slider oscillates back and forth with the slider, the change of impulse moment in a macroimpact process is more intense, and there are multiple secondary impacts in a macroimpact 


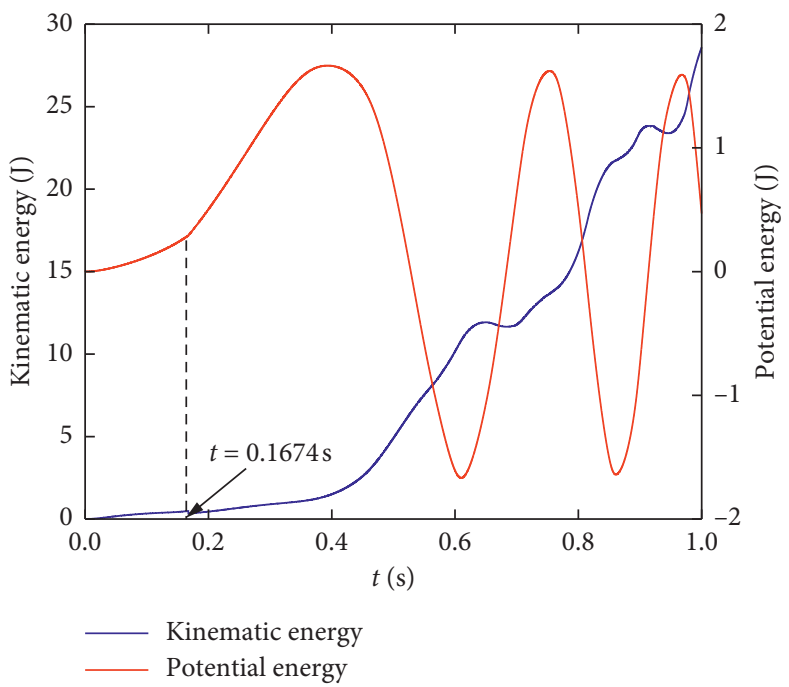

FIGURE 9: Energy variation of the system.

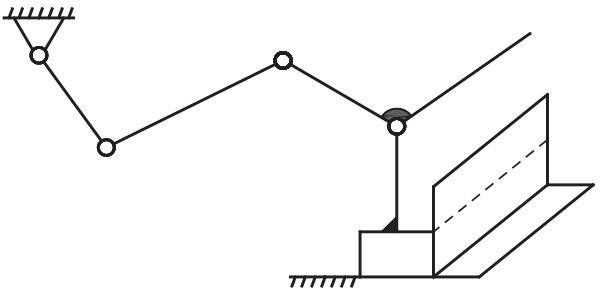

(a)

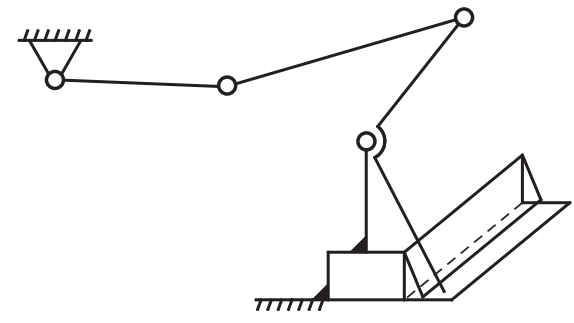

(b)

Figure 10: The planar double-folded metamorphic mechanism. (a) Configuration 1. (b) Configuration 2.

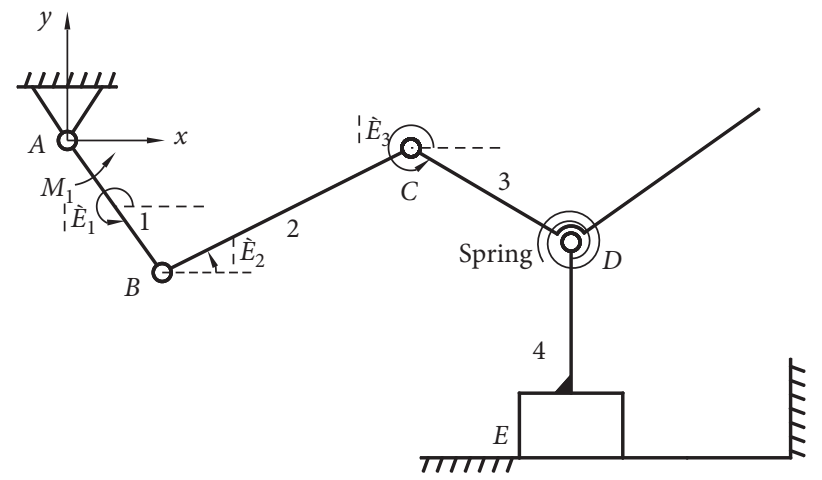

FIgURE 11: The schematic diagram of the planar double-folded metamorphic mechanism.

TABLE 2: Geometric and inertia parameters of the planar double-folded metamorphic mechanism.

\begin{tabular}{lccc}
\hline Parameter & Value & Parameter & \\
\hline$L_{A B}(\mathrm{~m})$ & 0.08 & $L_{B C}(\mathrm{~m})$ & Value \\
$L_{C D}(\mathrm{~m})$ & 0.09 & $L_{A E y}(\mathrm{~m})$ & 0.2 \\
$m_{1}(\mathrm{~kg})$ & 0.075 & $m_{2}(\mathrm{~kg})$ & 0.1425 \\
$m_{3}(\mathrm{~kg})$ & 0.162 & $m_{4}(\mathrm{~kg})$ & 0.165 \\
$J_{1}\left(\mathrm{~kg} \cdot \mathrm{m}^{2}\right)$ & $6.3818 \times 10^{-5}$ & $J_{B C}\left(\mathrm{~kg} \cdot \mathrm{m}^{2}\right)$ & 0.455 \\
$J_{D C}\left(\mathrm{~kg} \cdot \mathrm{m}^{2}\right)$ & $8.83 \times 10^{-4}$ & $k(\mathrm{~N} \cdot \mathrm{m} / \mathrm{rad})$ & $2.32 \times 10^{-3}$ \\
$c(\mathrm{~N} \cdot \mathrm{m} /(\mathrm{rad} / \mathrm{s}))$ & $0.18 / \pi$ & $\theta_{0}(\mathrm{rad})$ & $3.24 / \pi$ \\
\hline
\end{tabular}

Note. $L_{A B}$ is the corresponding link length between joints $A$ and $B, L_{B C}$ is the corresponding link length between joints $B$ and $C, L_{C D}$ is the corresponding link length between joints $C$ and $D, L_{A E y}$ is the corresponding distance along the $y$-axis between joints $A$ and $E, m_{i}(i=1,2,3,4)$ is the mass of component $i, J_{1}$ is the moment of inertia of component 1 around the center of mass, $J_{B C}$ is the moment of inertia of component 2 around the joint $C$, $J_{D C}$ is the moment of inertia of component 3 around the joint $C, k$ is the spring constant, $c$ is the damping constant, and $\theta_{0}$ is the initial angle of the spring. 


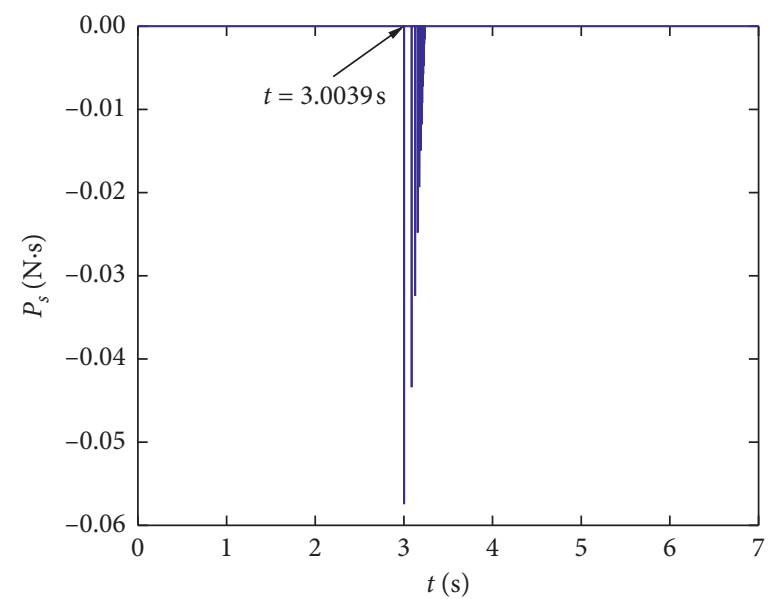

FIGURE 12: The impulse moment in the whole simulation process.

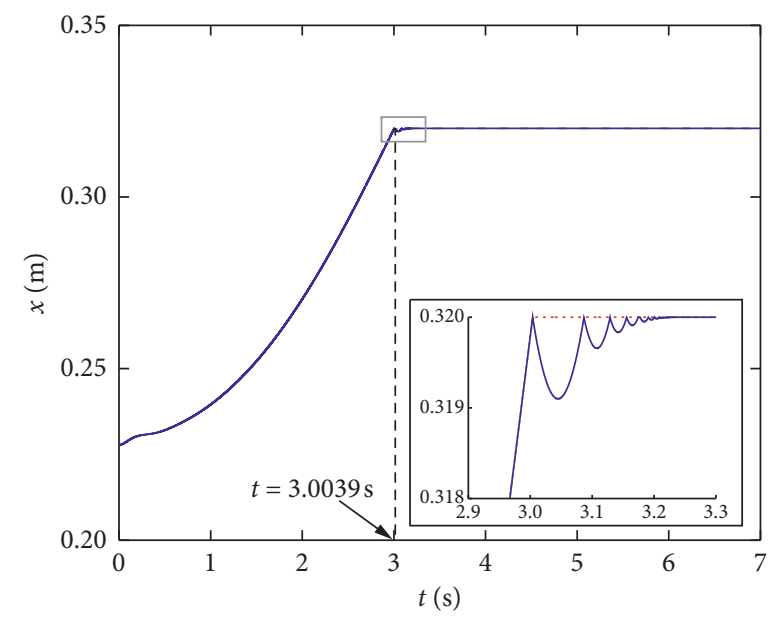

- - Without considering impact motion

— Considering impact motion

(a)

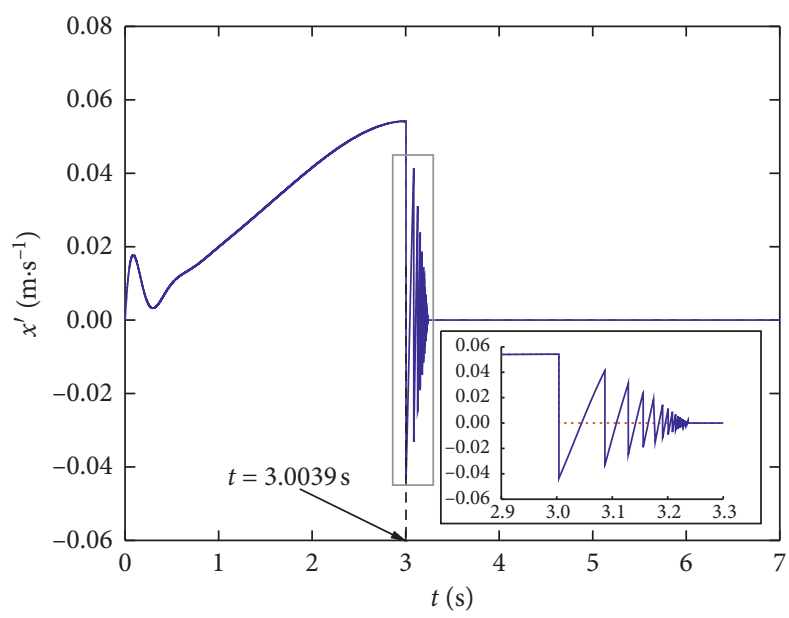

- - - Without considering impact motion — Considering impact motion

(b)

Figure 13: (a) Position variation of the slider. (b) Velocity variation of the slider.

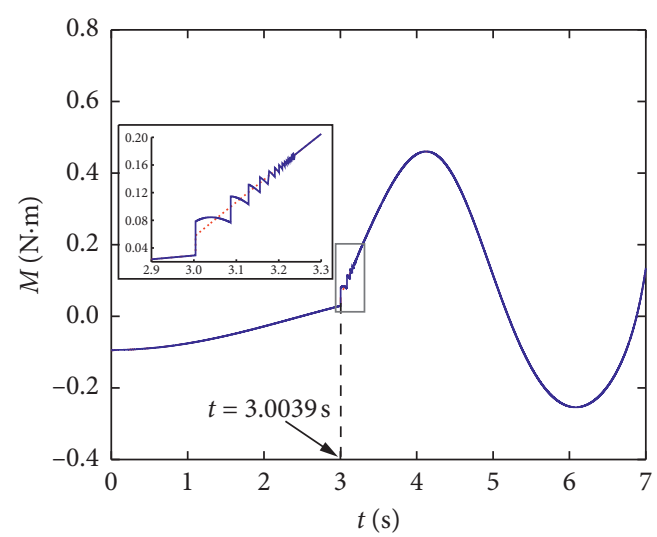

- Without considering impact motion Considering impact motion

Figure 14: Driven moment variation.

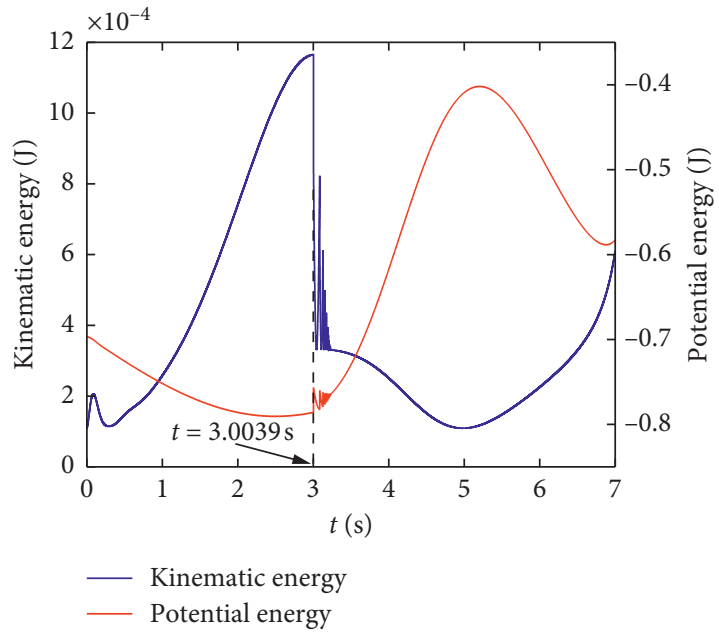

FIGURE 15: Driven moment variation. 


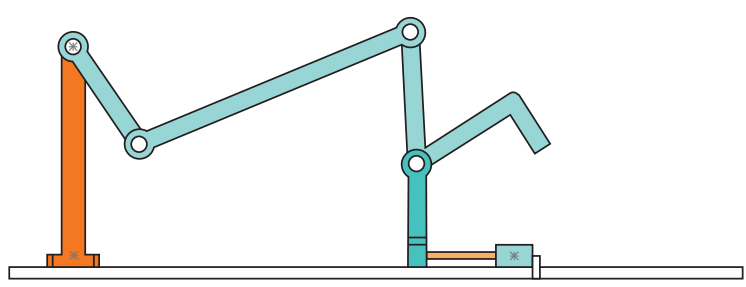

Figure 16: Three-dimensional graph.

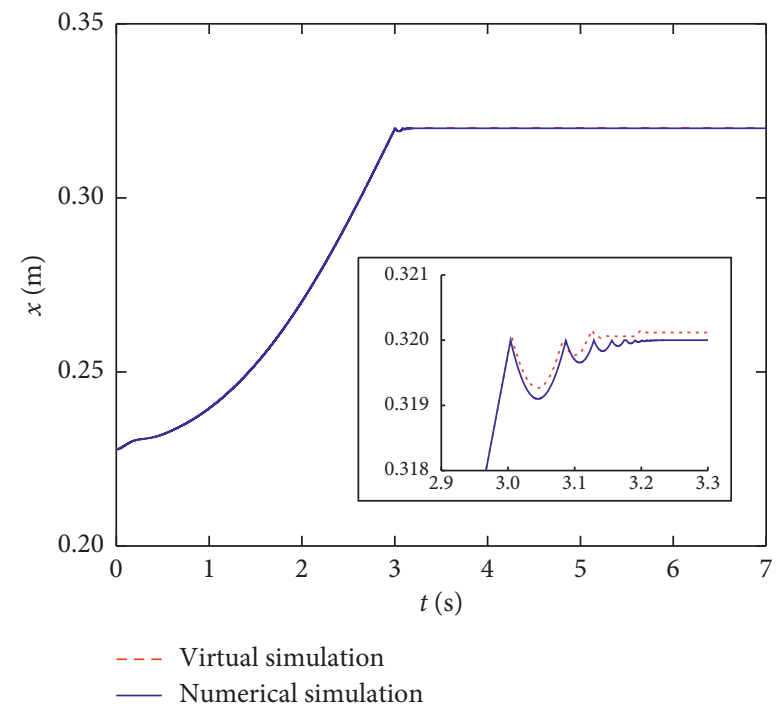

(a)

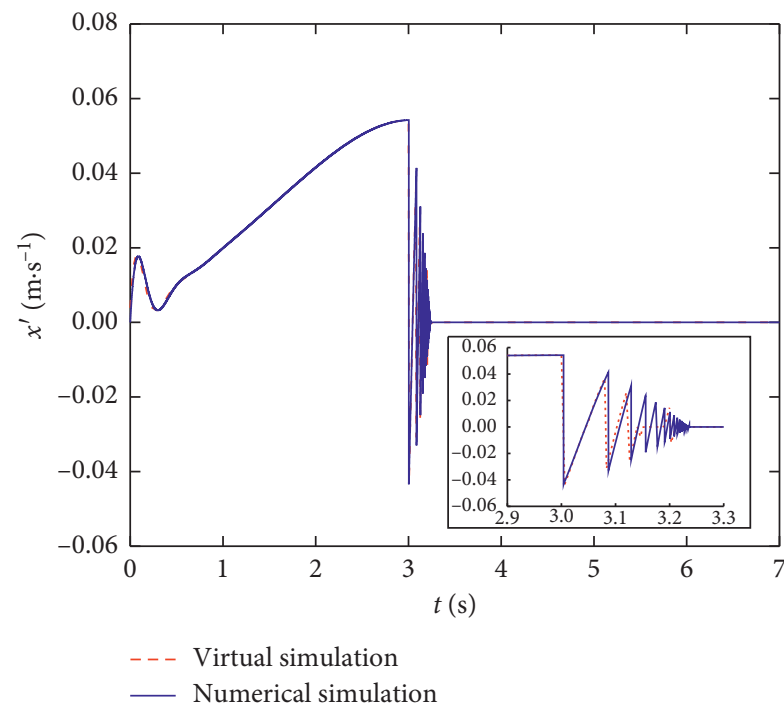

(b)

Figure 17: (a) Position variation of the slider. (b) Velocity variation of the slider.

process. In addition, the time interval of secondary impacts is becoming smaller and smaller. After several consecutive secondary impacts, the impulse moment of the slider becomes zero until the slider and the frame are completely merged, and the configuration transformation ends.

Figure 13 shows the variation of the slider position and slider velocity, respectively. From Figure 13, it can be seen that the impact motion considering the configuration transformation makes the slider velocity change complicatedly, and the change gradually decreases with time. After the slider and the frame are merged, velocity of the slider decreases to 0 and the mechanism enters the second configuration.

The variation of the driven moment is illustrated through Figure 14. It can be seen from the results in Figure 14 that the driven moment of the mechanism changes accordingly after the impact motion occurs. Comparing the displacement and velocity curves of the slider, the simulation results further illustrate the effectiveness of the model established.

Figure 15 shows the variation of dynamic behavior for the system in the whole simulation process from the perspective of energy. As shown in Figure 15, kinematic energy and potential energy of the system changes greatly at the instant of configuration transformation. This is caused by the impact of configuration transformation.
In addition, according to geometric and inertia parameters of the planar double-folded metamorphic mechanism in Table 2, a three-dimensional model is established in SolidWorks, as shown in Figure 16. The motion plug-in of the SolidWorks software is used to carry out dynamic virtual simulation on it. The results are shown in Figure 17 and are compared with the numerical calculation results shown in Figure 13.

From Figure 17, it can be found that the law of motion curve obtained by numerical calculation and virtual simulation is basically the same, but there is a slight deviation. This is due to the different calculation accuracy of MATLAB and SolidWorks at the time of configuration transformation.

\section{Conclusion}

The paper presents a new method of establishing impact dynamic models of the planar constrained metamorphic mechanism. Based on the dynamic modeling in the subconfiguration movement stage, the stable and the mobile impact dynamic equations of the planar constrained metamorphic mechanism are, respectively, established by considering the metamorphic forms and the constraint forms of the cutoff joint.

The stable and the mobile impulse solving models of the planar constrained metamorphic mechanism during 
configuration transformation are derived by the corresponding impact dynamic equation and the coefficient of restitution equation. The impulse and the generalized velocity increments are completely decoupled, which is beneficial to solve the abrupt change of the generalized velocity. Then, the system state variables after configuration transformation are calculated according to the initial conditions in configuration transformation, which provides an important basis and a precondition for the impact dynamic modeling of the planar constrained metamorphic mechanism and further improves its configuration-complete dynamic models.

Using the proposed method, the open-loop three-bar constrained metamorphic mechanism and the planar double-folded metamorphic mechanism are taken as examples to simulate the mobile impact motion and the stable impact motion, respectively. The results show, under the conditions of considering and ignoring the impact motion during configuration transformation, that the motion states of the open-loop system after configuration transformation are obviously different and the motion states of the closed-loop system during configuration transformation which are violent vibrations. In addition, the proposed impact dynamic modeling method of the planar constrained metamorphic mechanism not only provides a theoretical basis for our follow-up research work, but also can be applied to solve the internal collision dynamic problem of general planar robotic systems.

\section{Nomenclature}

$\operatorname{DOF}(\mathrm{s}): \quad$ Degree(s) of freedom

Superscript Configuration $p$

p:

${ }^{p} \mathbf{q}: \quad$ Independent generalized coordinate

$p$ D: $\quad$ Inertial matrix

${ }^{p} \mathbf{H}$ : $\quad$ Gravity, Coriolis, and centripetal force vector

${ }^{p} \tau_{a}: \quad$ Generalized force vector

$p_{\tau_{c}}$ : $\quad$ Generalized elastic and other generalized force vector

${ }^{p} X_{i}: \quad$ Position of the impact point $i$

${ }^{p} \varphi_{f_{(i)}}$ : $\quad$ Position function of the impact point $i$ in the multibody system $f_{(i)}$

$P\left[\mathbf{J}_{\mathbf{q}_{f_{(i)}}^{v}}^{v}\right]: \quad$ First-order kinematic influence coefficient between the impact point $i$ and generalized velocity in the multibody system $f_{(i)}$

${ }^{p} \mathbf{q}_{f_{(i)}}$ : Independent generalized coordinate of the multibody system $f_{(i)}$

$p \mathbf{D}_{f_{(i)}}$ : $\quad$ Inertial matrix of the multibody system $f_{(i)}$

${ }^{p} \mathbf{H}_{f_{(i)}}$ : $\quad$ Gravity, Coriolis, and centripetal force vector of the multibody system $f_{(i)}$

${ }^{p} \tau_{a f_{(i)}}$ : Generalized force vector of the multibody system $f_{(i)}$

${ }^{p} \tau_{c f_{(i)}}: \quad$ Generalized elastic and other generalized force vector of the multibody system $f_{(i)}$

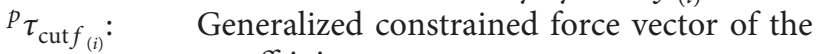
cutoff joint

${ }^{p} \tau_{s f}: \quad \quad$ Generalized stable impact force vector of the multibody system $f$
$P\left[\mathbf{J}_{\mathbf{q}_{f(i)}}^{\text {cut }}\right]: \quad$ First-order kinematic influence coefficient between the cutoff joint and generalized velocity in the multibody system $f_{(i)}$

${ }^{p} F_{\text {cut } f_{(i)}}: \quad$ Constraint force at the cutoff joint in the multibody system $f_{(i)}$

${ }^{P} F_{s}: \quad$ Stable impact force

${ }^{p} \mathbf{D}_{\text {cut }}$ : The coefficient matrix of ${ }^{p} F_{\text {cut } f_{(i)}}$ with respect to $p \ddot{q}$

$p \mathbf{H}_{\text {cut } f_{i}:}$ A column matrix

${ }^{p} \mathbf{D}_{\text {cut } f}$ : The coefficient matrix of ${ }^{p} \mathbf{F}_{\text {cut } f_{(i)}}$ with respect to $p \ddot{\mathbf{q}}_{f(i)}$

$p_{\tau_{m f^{(i)}}}: \quad$ Generalized mobile impact force vector of the multibody system $f_{(i)}$

$p F_{m_{(i)}}$ : $\quad$ Mobile impact force of the multibody system $f_{(i)}$

$v_{i}$ : $\quad$ Velocity of impact point relative to the inertial coordinate system before configuration transformation

$\Delta v_{i}: \quad$ Velocity increments immediately after configuration transformation

$n$ : $\quad$ Normal vector of impact point velocity increment

$e: \quad$ Coefficient of restitution

$p \mathbf{P}_{s}$ : $\quad$ Stable impulse at the impact point

${ }^{p} \mathbf{P}_{m_{(i)}}: \quad$ Mobile impulse at the impact point.

\section{Data Availability}

All data generated or analyzed during this study are included in this published article, and other pieces of information are available from the corresponding author upon reasonable request.

\section{Conflicts of Interest}

The authors declare that there are no conflicts of interest regarding the publication of this paper.

\section{Acknowledgments}

This study was partly supported by the National Natural Science Foundation of China (nos. 51275352 and 51475330), Natural Science Foundation of Tianjin (nos. 17JCQNJC03900 and 18JCQNJC05300), Tianjin Municipal Education Commission Research Program (2018KJ205), and Program for Innovative Research Team in University of Tianjin (TD13-5037).

\section{References}

[1] W. Zhang, X. Ding, and J. Liu, "A representation of the configurations and evolution of metamorphic mechanisms," Mechanical Sciences, vol. 7, no. 1, pp. 39-47, 2016.

[2] J. S. Dai and J. Rees Jones, "Mobility in metamorphic mechanisms of foldable/erectable kinds," Journal of $\mathrm{Me}$ chanical Design, vol. 121, no. 3, pp. 375-382, 1999.

[3] C. Valsamos, V. Moulianitis, and N. Aspragathos, "Index based optimal anatomy of a metamorphic manipulator for a given task," Robotics and Computer-Integrated Manufacturing, vol. 28, no. 4, pp. 517-529, 2012. 
[4] C. Valsamos, V. C. Moulianitis, A. I. Synodinos, and N. A. Aspragathos, "Introduction of the high performance area measure for the evaluation of metamorphic manipulator anatomies," Mechanism and Machine Theory, vol. 86, pp. 88-107, 2015.

[5] K. Xu, L. Li, S. Bai, Q. Yang, and X. Ding, "Design and analysis of a metamorphic mechanism cell for multistage orderly deployable/retractable mechanism," Mechanism and Machine Theory, vol. 111, pp. 85-98, 2017.

[6] W. Zhang, F. Liu, Y. Lv, and X. Ding, "Design and analysis of a metamorphic mechanism for automated fibre placement," Mechanism and Machine Theory, vol. 130, pp. 463-476, 2018.

[7] F. Liu, W. Zhang, K. Xu, H. Deng, and X. Ding, "A planar mechanism with variable topology for automated fiber placement," in Proceedings of the 2018 International Conference on Reconfigurable Mechanisms and Robots (ReMAR), June 2018.

[8] V. Charalampos, V. C. Moulianitis, and N. A. Aspragathos, "Kinematic synthesis of structures for metamorphic serial manipulators," Journal of Mechanisms and Robotics, vol. 6, no. 4, Article ID 041005, 2014.

[9] D. Gan, J. Dias, and L. Seneviratne, "Unified kinematics and optimal design of a 3rRPS metamorphic parallel mechanism with a reconfigurable revolute joint," Mechanism and $M a$ chine Theory, vol. 96, pp. 239-254, 2016.

[10] S. Li, H. Wang, X. Li, H. Yang, and J. S. Dai, “Task-orientated design method of practical constraint metamorphic mechanisms," Journal of Mechanical Engineering, vol. 54, no. 3, pp. 26-35, 2018.

[11] P. Martín A, A. Butti, V. Tamellini, A. Cardona, and L. Ghezzi, "Topological synthesis of planar metamorphic mechanisms for low-voltage circuit breakers," Mechanics Based Design of Structures and Machines, vol. 40, no. 4, pp. 453-468, 2012.

[12] S. Li, H. Wang, Q. Meng, and J. S. Dai, “Task-based structure synthesis of source metamorphic mechanisms and constrained forms of metamorphic joints," Mechanism and Machine Theory, vol. 96, pp. 334-345, 2016.

[13] G. Gilardi and I. Sharf, "Literature survey of contact dynamics modelling," Mechanism and Machine Theory, vol. 37, no. 10, pp. 1213-1239, 2002.

[14] Y. C. Duan and D. G. Zhang, "Rigid-flexible coupling dynamics of a flexible robot with impact," Advanced Materials Research, vol. 199-200, pp. 243-250, 2011.

[15] G. Jin, G. Wu, B. Chang, and L. Chen, "Dynamic analysis of manipulator with contact impact," Transactions of the Chinese Society for Agricultural Machinery, vol. 47, pp. 369-375, 2016.

[16] Q.-H. Dong and L. Chen, "Impact dynamics analysis of freefloating space manipulator capturing satellite on orbit and robust adaptive compound control algorithm design for suppressing motion," Applied Mathematics and Mechanics, vol. 35, no. 4, pp. 413-422, 2014.

[17] Z. Deng, Q. Wang, Q. Quan, S. Jiang, and D. Tang, "Impact dynamics of a differential gears based underactuated robotic arm for moving target capturing," Mechatronics, vol. 40, pp. 208-219, 2016.

[18] J. Choi, H. Ryu, and B. Yi, "Variable impact dynamics of a finger mechanism," in Proceedings of the 2011 8th International Conference on Ubiquitous Robots and Ambient Intelligence, pp. 23-26, Incheon, South Korea, November 2011.

[19] M. Machado, P. Moreira, P. Flores, and H. M. Lankarani, "Compliant contact force models in multibody dynamics: evolution of the Hertz contact theory," Mechanism and Machine Theory, vol. 53, pp. 99-121, 2012.
[20] D. Bhalerao and S. Anderson, "Modeling intermittent contact for flexible multibody systems," Nonlinear Dynamics, vol. 60, no. 1-2, pp. 63-79, 2010.

[21] M. Ahmadizadeh, A. M. Shafei, and M. Fooladi, "A recursive algorithm for dynamics of multiple frictionless impact-contacts in open-loop robotic mechanisms," Mechanism and Machine Theory, vol. 146, pp. 1-20, 2020.

[22] M. H. Korayem and M. Taheri, "Modeling of various contact theories for the manipulation of different biological micro/ nanoparticles based on AFM," Journal of Nanoparticle Research, vol. 16, no. 1, pp. 1-13, 2014.

[23] M. H. Korayem, H. Khaksar, M. Taheri et al., "Modeling of contact theories for the manipulation of biological micro/ nanoparticles in the form of circular crowned rollers based on the atomic force microscope," Journal of Applied Physics, vol. 114, no. 18, Article ID 183715, 2013.

[24] P. Flores and J. Ambrósio, "On the contact detection for contact-impact analysis in multibody systems," Multibody System Dynamics, vol. 24, no. 1, pp. 103-122, 2010.

[25] J. Y. Wang, Z. Y. Liu, and J. Z. Hong, "Partition method and experimental validation for impact dynamics of flexible multibody system," Acta Mechanica Sinica, vol. 34, no. 3, pp. 482-492, 2018.

[26] Y. Hurmuzlu and D. B. Marghitu, "Rigid body collisions of planar kinematic chains with multiple contact points," The International Journal of Robotics Research, vol. 13, no. 1, pp. 82-92, 1994.

[27] A. M. Shafei and H. R. Shafei, "Dynamic modeling of planar closed-chain robotic manipulators in flight and impact phases," Mechanism and Machine Theory, vol. 126, pp. 141154, 2018.

[28] H. Gattringer, H. Bremer, and M. Kastner, "Efficient dynamic modeling for rigid multi-body systems with contact and impact," Acta Mechanica, vol. 219, no. 1-2, pp. 111-128, 2011.

[29] R. Wang, H. Chen, and J. Dai, "Dynamic stability study of a novel controllable metamorphic palletizing robot mechanism," Journal of Mechanical Engineering, vol. 13, no. 19, pp. 39-47, 2017.

[30] L. Bruzzone and G. Bozzini, "A flexible joints microassembly robot with metamorphic gripper," Assembly Automation, vol. 30, no. 3, pp. 240-247, 2010.

[31] Y. Song, X. Ma, and J. S. Dai, "A novel 6R metamorphic mechanism with eight motion branches and multiple furcation points," Mechanism and Machine Theory, vol. 142, pp. 1-19, 2019.

[32] W. Ye, Y. Fang, K. Zhang, and S. Guo, "Mobility variation of a family of metamorphic parallel mechanisms with reconfigurable hybrid limbs," Robotics and Computer-Integrated Manufacturing, vol. 41, pp. 145-162, 2016.

[33] G. Jin, X. Ding, and Q. Zhang, "Research on configurationcomplete dynamics modeling and numerical simulation of metamorphic mechanism," Acta Aeronautica et Astronautica Sinica, vol. 25, pp. 401-405, 2004.

[34] S. Yang, G. Jin, J. Yun, and Z. Feng, "Research on impact motion in metamorphic mechanism," China Mechanical Engineering, vol. 20, pp. 1608-1612, 2009.

[35] K. S. Anderson and J. H. Critchley, "Improved "order-N" performance algorithm for the simulation of constrained multi-rigid-body dynamic systems," Multibody System Dynamics, vol. 9, no. 2, pp. 185-212, 2003.

[36] J. Hong, Computational Dynamics of Multibody Systems, High Education Press, Beijing, China, 1999.

[37] Q. Yang, H. Wang, S. Li, and J. S. Dai, “Type synthesis of constrained metamorphic mechanisms with structural forms 
of metamorphic joints," Journal of Mechanical Engineering, vol. 50, no. 13, pp. 1-8, 2014.

[38] B. Chang, G. Jin, and J. S. Dai, "Type synthesis of metamorphic mechanism based on variable constraint screw theory," Journal of Mechanical Engineering, vol. 50, no. 5, pp. 17-25, 2014. 